\title{
Verbreitung und betriebliche Bestimmungsfaktoren von Arbeitszeitkonten ${ }^{* *}$
}

\section{Zusammenfassung}

Arbeitszeitkonten ermöglichen eine variable Arbeitszeitverteilung in Betrieben, womit sich Nachfrageschwankungen abfedern und der Arbeitseinsatz an das Arbeitsaufkommen anpassen lassen. Im Zuge der Finanz- und Wirtschaftskrise 2008/09 wurden sie umfangreich genutzt, um Arbeitszeiten vorübergehend zu verkürzen. Vor diesem Hintergrund haben sie als Instrument zur Arbeitszeitflexibilisierung einen neuen Aufschwung in der öffentlichen und wissenschaftlichen Diskussion erfahren. Damit stellt sich die Frage nach den Bestimmungsfaktoren von Arbeitszeitkonten. Mit Daten des Betriebspanels des Instituts für Arbeitsmarkt- und Berufsforschung und logistischen sowie OLS-Regressionen wird untersucht, welche Faktoren das Auftreten von Arbeitszeitkonten in Betrieben und die Verbreitungsquote bei Beschäftigten erklären. Die industriellen Beziehungen sind ein wesentlicher Bestimmungsfaktor für Arbeitszeitkonten in Betrieben. Ebenfalls steigt mit einem zunehmenden Anteil an Leiharbeitern und qualifizierten Beschäftigten die Wahrscheinlichkeit von Arbeitszeitkonten, während sie mit zunehmendem Anteil befristet Beschäftigter sinkt. Die Verbreitungsquote bei Beschäftigten hängt positiv mit einem Betriebs-/Personalrat sowie dem Anteil an Leiharbeitern und negativ mit dem Anteil befristet Beschäftigter zusammen.

Schlagwörter: Arbeitszeit, Arbeitszeitflexibilisierung, Arbeitszeitkonten, Work-Life Balance

\section{Distribution of working-time accounts and its establishment-specific determinants}

\begin{abstract}
Working-time accounts offer variable distributions of hours worked in establishments. Using working-time accounts allows companies to absorb fluctuations in demand and adjust the work effort to the workload. During the financial and economic crisis of 2008/09 establishments used working-time accounts to temporarily reduce working hours. Against this background, working-time accounts have
\end{abstract}

* Dr. Ines Zapf, Wissenschaftliche Mitarbeiterin am Institut für Arbeitsmarkt- und Berufsforschung, Forschungsbereich „Prognosen und gesamtwirtschaftliche Analysen“, Regensburger Straße 104, D-90478 Nürnberg. E-Mail: ines.zapf@iab.de

** Artikel eingegangen: 29.04.2016, revidierte Fassung nach doppelt-blindem Begutachtungsverfahren akzeptiert: 04.12.2017 
become a topic of interest and discussion in the public and scientific domains. For this reason, questions have arisen about determinants that influence working-time accounts. Drawing on data from the company survey panel collected by the Institute for Employment Research and applying logistic and OLS-regression models, the article examines working-time account determinants and the distribution of such accounts among employees. We outline how industrial relations factors play an important role in influencing working-time accounts in establishments. In addition, we contend that an increasing share of agency workers and of qualified employees raises the probability that working-time accounts are used, whereas an increase in the share of employees on fixed-term contracts is likely to decrease. Further, whilst the distribution among employees is positively associated with the presence of a works council and agency workers; it is negatively associated with the share of employees on fixed-term contracts.

Key words: Working time, working-time flexibility, working-time accounts, work-life balance (JEL: J28, J81)

\section{Einleitung}

Die deutsche Wirtschaft wurde durch die Finanz- und Wirtschaftskrise 2008/2009 schwer getroffen. Dennoch erwies sich der Arbeitsmarkt als robust und die Beschäftigungsverluste waren geringer als angenommen. Diese überraschende Entwicklung lässt sich zum einen auf die intensive Nutzung von internen Flexibilitätsinstrumenten zurückführen. Dabei haben Betriebe solche Instrumente genutzt, auf die sie bereits in Nicht-Krisenphasen zurückgegriffen haben (Kümmerling \& Lehndorff, 2014). Zum anderen haben Betriebe Arbeitskräfte noch stärker als in der Krise zu Beginn der 2000er gehortet (Bosch, 2011). Um Arbeitskräfte zu horten, kann entweder die Arbeitszeit temporär verkürzt oder die Arbeitsintensität und damit die Arbeitsproduktivität verringert werden (Herzog-Stein \& Zapf, 2014). Während der Krise wurden die Arbeitszeiten der Beschäftigten durch zwei unterschiedliche Formen von Instrumenten deutlich reduziert. Erstens durch die Kurzarbeit mit ihrem spezifischen institutionellen Hintergrund und öffentlicher Förderung und zweitens durch Arbeitszeitanpassungen ohne öffentliche finanzielle Unterstützung (Kümmerling \& Lehndorff, 2014). Ein Beispiel dafür sind Arbeitszeitkonten, die in vielen Betrieben zur Verfügung standen. Vor diesem Hintergrund hat die Forschung zu Arbeitszeitkonten als Instrument zur Arbeitszeitflexibilisierung einen neuen Aufschwung genommen (vgl. z.B. Herzog-Stein \& Seifert, 2010; Bellmann \& Gerner, 2011; Boeri \& Brücker, 2011; Burda \& Hunt, 2011; Bellmann, Gerner \& Upward, 2012; Kümmerling \& Lehndorff, 2014; Bellmann \& Hübler, 2015; Balleer, Gehrke \& Merkl, 2017).

Arbeitszeitkonten gelten als zentrales Instrument, um flexible oder von der vertraglichen Wochenarbeitszeit abweichende Arbeitszeiten zu verwalten und $\mathrm{zu}$ dokumentieren und damit Nachfrageschwankungen abzufedern (vgl. z.B. Seifert, 1996; Hildebrandt, 2006; Groß \& Schwarz, 2010; Lindecke, 2015). Indem Arbeitszeiten an das Arbeitsaufkommen angepasst werden kann (zumindest vorübergehend) auf eine Variation der Beschäftigtenzahl durch Einstellungen und Entlassungen verzichtet werden. Ebenso lassen sich Leerlaufzeiten vermeiden, der Arbeitseinsatz effizienter gestalten und die Produktivität steigern (Munz, Bauer \& Groß, 2002). Mit Arbeitszeitkonten ergeben sich für die Betriebe auch Kostenvorteile, da bezahlte Überstunden verringert oder vermieden werden können. Durch 
die beschäftigungssichernde Wirkung sind auch Gewerkschaften und Betriebs- oder Personalräte sowie Arbeitnehmer an Arbeitszeitkonten interessiert. Im Zuge des Tauschs von Arbeitszeitflexibilität gegen Beschäftigungssicherheit spielen sie in Bündnissen für Arbeit eine wichtige Rolle (Ludewig, 2001). Darüber hinaus können Gewerkschaften, Betriebs-/ Personalräte und Beschäftigte an Arbeitszeitkonten interessiert sein, um eine höhere Zeitsouveränität zu realisieren. Indem Arbeitszeiten nach individuellen Bedürfnissen variiert werden, erreichen Beschäftigte eine bessere Vereinbarkeit von Beruf und Familie. Eine bessere Balance zwischen beruflichen und privaten Zeitanforderungen ist aber auch für die Betriebe vorteilhaft, da Beschäftigte zufriedener und engagierter sind und insgesamt die Attraktivität als Arbeitgeber steigen kann (Goudswaard et al., 2012).

Prinzipiell bieten also Arbeitszeitkonten die Chance einer ,Win-Win-Situation“ für Betriebe und Beschäftigte. Jedoch kommt es aufgrund unterschiedlicher Interessen und Motiven zu Konflikten zwischen Arbeitgebern und Arbeitnehmern (Kümmerling \& Lehndorff, 2014). Häufig stehen die betrieblichen Bedürfnisse, wie Konjunkturanpassung, den individuellen Bedürfnissen der Beschäftigten, wie eine höhere Zeitautonomie, gegenüber. So richtet sich der Aufbau von Guthaben auf Arbeitszeitkonten häufig primär nach den Markterfordernissen. Auch haben Betriebe Steuerungsmöglichkeiten beim Abbau der Zeitguthaben. Ein beabsichtigter Freizeitausgleich ist meist mit den Vorgesetzten und Kollegen abzusprechen (Bosch, Ellguth \& Promberger, 1992; Bosch, 1996; Promberger, Böhm, Heyder, Pamer \& Strauß, 2002), womit die antizipierte Zeitsouveränität der Beschäftigten eingeschränkt wird. Bei einer ausschließlichen Orientierung an betriebliche Markterfordernisse gehen aber Betriebe das Risiko einer sinkenden Motivation und Loyalität der Beschäftigten ein (Goudswaard et al., 2012) und es kann zu einer inneren Kündigung der Beschäftigten kommen.

Im Zuge des Aufbaus von Guthaben auf Arbeitszeitkonten leisten Beschäftigte transitorische Überstunden, also solche, die zu einem späteren Zeitpunkt wieder in Freizeit ausgeglichen werden. Zum Zeitpunkt des Leistens von transitorischen Überstunden ist aber nicht immer klar, ob diese später tatsächlich abgebaut werden. Zum einen können transitorische Überstunden zu bezahlten Überstunden werden, wenn Beschäftigte die mehr geleistete Arbeitszeit in einem bestimmten, vorab festgelegten Zeitraum nicht ausgleichen können und Betriebe die mehr geleisteten Stunden schließlich ausbezahlen. Zum anderen können transitorische Überstunden zu unbezahlten Überstunden werden, wenn sie nicht rechtzeitig ausgeglichen und zu bestimmten Stichtagen gekappt werden.

Insgesamt bieten Arbeitszeitkonten damit nicht nur Chancen und Vorteile, sondern bergen auch Risiken und Nachteile für Betriebe und Beschäftigte (vgl. dazu ausführlicher auch Zapf 2016). Dennoch führen Betriebe Arbeitszeitkonten ein, um transitorische Überstunden und den vorgesehenen Freizeitausgleich zu dokumentieren. Diese transitorischen Überstunden sind inzwischen weit verbreitet und stehen mit der Zunahme von Arbeitszeitkonten in Zusammenhang.

Vor diesem Hintergrund stellt sich die Frage, welche treibenden Kräfte es für die Verbreitung von Arbeitszeitkonten gibt. Welche betrieblichen Faktoren begünstigen Arbeitszeitkonten und welche erschweren sie? Welche Rolle spielen dabei die industriellen Beziehungen und die Beschäftigungsstruktur in einem Betrieb? Der vorliegende Beitrag geht diesen Fragen nach und stellt die betriebliche Verbreitung sowie die Bestimmungsfaktoren von 
Arbeitszeitkonten im Zeitverlauf dar. Darüber hinaus wird die Verbreitungsquote von Arbeitszeitkonten bei Beschäftigten in Betrieben mit Arbeitszeitkonten analysiert, um den Blick auf Arbeitszeitkonten zu erweitern.

Der Aufsatz ist wie folgt gegliedert: Nach einem kurzen Überblick über den Forschungsstand (Abschnitt 2) werden die verschiedenen Modelle und Regulierungsmöglichkeiten von Arbeitszeitkonten aufgezeigt (Abschnitt 3). Darauf folgend werden Hypothesen zu den betrieblichen Bestimmungsfaktoren von Arbeitszeitkonten (Abschnitt 4) und die in den Analysen verwendeten Daten, Variablen und Methode vorgestellt (Abschnitt 5). Abschnitt 6 stellt die deskriptiven und multivariaten Ergebnisse dar. Der Aufsatz schließt mit einer kurzen Zusammenfassung und zeigt die aktuellen gewerkschaftlichen Forderungen sowie die zukünftigen betrieblichen Herausforderungen in Bezug auf Arbeitszeitkonten auf (Abschnitt 7).

\section{Forschungsstand}

In der öffentlichen und wissenschaftlichen Diskussion werden Arbeitszeitflexibilität und die verschiedenen Instrumente zur Arbeitszeitflexibilisierung ambivalent gesehen. Zeit gilt als ein wesentlicher Bestimmungsfaktor, um Beruf und Privatleben zu vereinbaren. Dabei können flexible und autonome Arbeitszeiten individuelle Gestaltungsspielräume eröffnen und Beschäftigte dazu befähigen, ihre Arbeit mit ihren außerberuflichen Verantwortungen und Aktivitäten in Balance zu bringen (Hill, Hawkins, Ferris \& Weitzman, 2001; Wotschack, 2012; Lott, 2014). So können beispielsweise Beschäftigte mit kranken Kindern oder pflegebedürftigen Angehörigen bei variablen Arbeitszeiten besser in der Lage sein, ihren beruflichen Tätigkeiten nachzugehen. Stress wird vermindert oder vermieden, wenn Beschäftigte nicht zu fest vorgegebenen Zeiten am Arbeitsplatz sein müssen. Ergebnisse von Hunt (2013) weisen in diesem Kontext darauf hin, dass Beschäftigte mit flexiblen Arbeitszeiten tatsächlich eine höhere Freizeitzufriedenheit, aber eine geringere Arbeitszeitzufriedenheit haben. Allerdings kann nicht bestätigt werden, dass flexible Arbeitszeiten familienfreundlich sind. Die Ergebnisse beziehen sich allerdings nur auf Vollzeitbeschäftigte zwischen 20 und 54 Jahren in Westdeutschland.

Mit einem abnehmenden Regulierungsgrad von Arbeitszeiten steigt das Risiko, dass flexible Arbeitszeiten überwiegend von betrieblichen Interessen dominiert werden (Wotschack, 2012). So konstatiert Hildebrandt (2006), dass eine Flexibilisierung der Arbeitszeiten oft marktgetrieben, d.h. an den Bedürfnissen der Betriebe ausgerichtet ist. Dabei kann angenommen werden, dass eine hohe und nicht vorhersehbare Variabilität der Arbeitszeit sowie ein mangelnder Gestaltungseinfluss der Beschäftigten negativ auf die Gesundheit und Vereinbarkeit von Beruf und Familie wirken (Goudswaard et al., 2012). Flexible Arbeitszeiten können auch zu Überstunden und Arbeitsintensivierung führen (Gregory \& Milner, 2009), in deren Folge das Risiko von Arbeitsunfällen steigt (Allan, Brosnan \& Walsh, 1998; Looise, Van Riemsdijk \& De Lange, 1998; Goudswaard, Oeij, Brugman \& de Jong, 2009). Lehnen Betriebe arbeitnehmerfreundliche Arbeitszeitarrangements ab, kann dies die Motivation der Beschäftigten negativ beeinflussen und zu mehr Absentismus und Unpünktlichkeit führen. Beschäftigte könnten sich weniger auf berufliche Aufgaben konzentrieren 
und häufiger nach neuen Beschäftigungsmöglichkeiten suchen. Hingegen bieten arbeitnehmerfreundliche Arrangements die Chance, die Außenwirkung des Unternehmens bei (potenziellen) Kunden und in der Öffentlichkeit zu verbessern und so auch Umsatzsteigerungen zu erzielen. Auch kann die Arbeitgeberattraktivität gesteigert werden, infolgedessen betriebliche Rekrutierungs- und Weiterbildungskosten vermindert werden können (Goudswaard et al., 2012).

Der Bedarf nach einer marktorientierten Arbeitszeitflexibilität auf der einen Seite und der Vereinbarkeit von Beruf und Familie auf der anderen Seite stellt die Betriebe bei der Organisation von Arbeitszeiten vor große Herausforderungen. Eine Möglichkeit die unterschiedlichen Interessenlagen flexibler Arbeitszeiten zu berücksichtigen sind Arbeitszeitkonten, womit prinzipiell auf betriebliche und individuelle Bedürfnisse von Beschäftigten eingegangen werden kann. Die Entscheidung zur Einführung von Arbeitszeitkonten liegt auf der Arbeitgeberseite, wobei einige Betriebsmerkmale eine Einführung und Etablierung begünstigen und andere nicht. So zeigt Ludewig (2001) mit den Betriebspaneldaten des Instituts für Arbeitsmarkt- und Berufsforschung (IAB), dass Arbeitszeitkonten in einem Betrieb positiv mit der Betriebsgröße, einem Betriebs-/Personalrat und dem Anteil qualifizierter Beschäftigter korrelieren. Negative, aber nur teils signifikante Zusammenhänge ergeben sich beim Frauenanteil, der Wochenarbeitszeit und dem Anteil von Teilzeitbeschäftigten. Bellmann und Gewiese (2004) weisen unter anderem auf signifikant positive Zusammenhänge zwischen der Einführung von Kurzzeitkonten und der Betriebsgröße, dem Angestellten- und Auszubildendenanteil, der Existenz eines Betriebsrates sowie der Tarifbindung hin. Negative Zusammenhänge ergeben sich beim Frauenanteil und unbezahlten Überstunden.

Im Kontext der Finanz- und Wirtschaftskrise untersuchen Boeri und Brücker (2011) sowie Bellmann, Gerner und Upward (2012) die Bestimmungsfaktoren von Arbeitszeitkonten ebenfalls mit den Daten des IAB-Betriebspanels. Herzog-Stein und Zapf (2014) nutzen die Betriebsrätebefragung des Wirtschafts- und Sozialwissenschaftlichen Instituts (WSI) in der Hans-Böckler-Stiftung und analysieren die betrieblichen Gründe des Arbeitszeitkonteneinsatzes zur Beschäftigungssicherung. Die Autoren zeigen, dass ein Rückgang von Aufträgen und Umsatz und die Gewerkschaftsstärke in einem Betrieb positiv mit Arbeitszeitkonten korrelieren. Negative Zusammenhänge ergeben sich beim Anteil der Frauen und der Hochqualifizierten. Einige Studien analysieren auch die Wirkung von Arbeitszeitkonten im Kontext der Finanz- und Wirtschaftskrise mit den Daten des IAB-Betriebspanels. So weisen Bellmann und Gerner (2011) auf einen Rückgang der Löhne in Betrieben ohne Arbeitszeitkonten hin, während es in Betrieben mit Arbeitszeitkonten keinen signifikant negativen Lohneffekt gibt. Die Ergebnisse stützen somit die These, dass Einkommen über den Konjunkturzyklus hinweg durch Arbeitszeitkonten geglättet werden können. Hinsichtlich der Beschäftigungsentwicklung finden die Autoren zwar Unterschiede zwischen Betrieben mit Arbeitszeitkonten und ohne Arbeitszeitkonten, allerdings sind diese nicht signifikant. Damit zeigt sich nur ein schwacher Beleg für den Einfluss von Arbeitszeitkonten auf die Beschäftigungsstabilität zwischen dem 1. Halbjahr 2008 und 2009. Gerner (2012) analysiert den Effekt von Arbeitszeitkonten auf die Entwicklung der Arbeitsproduktivität und geht damit der Frage nach, ob in Betrieben mit Arbeitszeitkonten eine stärkere Hortung von Arbeitskräften stattgefunden hat. Die Ergebnisse zeigen keine signifikanten Unterschiede und wei- 
sen darauf hin, dass zwar Betriebe mit Arbeitszeitkonten in erheblichem Maße Beschäftigte gehortet haben, Betriebe ohne Arbeitszeitkonten jedoch auch.

Bellmann und Hübler (2015) gehen der Frage nach, ob Betriebe von Arbeitszeitkonten profitieren. Betriebe mit Arbeitszeitkonten weisen eine höhere Produktivität und höhere Investitionen auf als Betriebe ohne Arbeitszeitkonten. Dagegen gibt es keinen signifikanten Einfluss von Arbeitszeitkonten auf die Löhne je Beschäftigten und dem Betriebsüberschuss. ${ }^{1}$ Zwar zeigt sich ein negativer Einfluss auf Gewinne, dieser ist aber weniger wahrscheinlich für Betriebe mit einem hohen Anteil qualifizierter Beschäftigter. Während der Finanz- und Wirtschaftskrise haben Arbeitszeitkonten aber dazu beigetragen GewinneinbuBen zu reduzieren. Balleer, Gehrke \& Merkl (2017) analysieren, ob und wie sich eine Umsatzveränderung auf die Einstellungs- und Entlassungsquoten in Betrieben mit Arbeitszeitkonten und ohne Arbeitszeitkonten auswirkt. Die Autoren finden keine signifikanten Unterschiede, d.h. Betriebe mit Arbeitszeitkonten und ohne Arbeitszeitkonten zeigen bedingt auf eine Umsatzveränderung ein ähnliches Einstellungs- und Entlassungsverhalten. Die Hypothese, dass Betriebe ohne Arbeitszeitkonten die Kurzarbeit substitutiv dazu nutzten kann empirisch nicht belegt werden. Betriebe mit Arbeitszeitkonten nutzten die Kurzarbeit mehr als Betriebe ohne Arbeitszeitkonten.

Zusammenfassend betrachtet liegen zu den betrieblichen Bestimmungsfaktoren von Arbeitszeitkonten und ihrer Wirkung bereits empirische Befunde vor. Hinsichtlich der in diesem Aufsatz betrachteten Frage zu den Bestimmungsfaktoren von Arbeitszeitkonten in Betrieben und der Verbreitungsquote bei Beschäftigten in Betrieben mit Arbeitszeitkonten sind Ergebnisse aber nur punktuell oder fehlen und zeigen keine Entwicklungen im Zeitverlauf. In diesem Beitrag wird deshalb insbesondere die zeitliche Dimension berücksichtigt, indem die Bestimmungsfaktoren für mehrere Zeitpunkte analysiert und miteinander verglichen werden. Ein Vergleich verschiedener Zeitpunkte gibt Aufschluss darüber, ob es Unterschiede in der Wirkung von Merkmalen über die Zeit gibt.

\section{Formen und Regulierung von Arbeitszeitkonten}

\subsection{Formen}

Mit dem Auf- und Abbau von Zeitguthaben und -schulden auf Arbeitszeitkonten variieren Beschäftigte Dauer, Lage und Verteilung der Arbeitszeit. Meist gibt es Ober- und Untergrenzen und es wird ein Zeitraum festgelegt, innerhalb dessen das Konto auszugleichen ist. Die Bezugsgröße ist die tariflich oder vertraglich vereinbarte Wochenarbeitszeit, die im Durchschnitt wieder erreicht werden muss (Seifert, 2001, 2005; Groß \& Schwarz, 2010).

Inzwischen gibt es viele unterschiedliche Arbeitszeitkontenmodelle, die sich nach verschiedenen Merkmalen gruppieren lassen. Gleitzeitkonten, Überstundenkonten und Bandbreiten- bzw. Korridormodelle gelten als Kurzzeitkonten mit einem Ausgleichszeitraum von bis zu einem Jahr. Ansparmodelle sind Langzeit- bzw. Lebensarbeitszeitkonten mit einem Ausgleichszeitraum von über einem Jahr (Seifert, 2001, 2005; Hildebrandt, 2006,

1 Die Autoren berechnen den Betriebsüberschuss (operating surplus) aus der Differenz zwischen Umsatz und vorläufigen Arbeitslöhnen. 
2007). ${ }^{2}$ Mit diesen Konten soll die Erwerbsarbeitszeit von Beschäftigten im Lebensverlauf umverteilt werden, indem längere Erwerbsunterbrechungen oder ein früherer Ruhestand ermöglicht werden. Langzeit- bzw. Lebensarbeitszeitkonten zielen im Gegensatz zu Kurzzeitkonten also nicht darauf ab, betriebliche Produktions- und Arbeitsschwankungen auszugleichen (Wotschack, 2012; Seifert, Kümmerling \& Riedmann, 2013). ${ }^{3}$ Solche Konten sind bislang noch die Ausnahme, denn nur rund 2 Prozent der Betriebe in Deutschland verfügen über Langzeitkonten. Mit zunehmender Betriebsgröße steigt jedoch auch ihr Verbreitungsgrad (Ellguth, Gerner \& Zapf, 2013; Seifert, Kümmerling \& Riedmann, 2013).

In vielen Betrieben gibt es Mischformen, verschiedene Arbeitszeitkontenmodelle für unterschiedliche Beschäftigtengruppen und für einige Beschäftigte werden auch mehrere Modelle nebeneinander geführt. So können Beschäftigte sowohl über ein Gleitzeit- als auch über ein Ansparkonto verfügen (Seifert, 2005; Hildebrandt, 2007). Dadurch lassen sich angesammelte und nicht rechtzeitig ausgeglichene Stunden des Kurzzeitkontos auf ein Langzeitkonto übertragen, womit eine monetäre Abgeltung oder Kappung der Zeitguthaben potenziell vermieden werden kann (Groß \& Schwarz, 2010). Dennoch weisen Groß und Schwarz (2006) darauf hin, dass angesammelte Guthaben nach einer Überschreitung von vereinbarten Obergrenzen (34\%) auch finanziell abgegolten werden $(6 \%)$ oder verfallen $(1 \%)$. Meist sollen sie aber möglichst bald in Freizeit ausgeglichen (12\%), in den nächsten Ausgleichszeitraum (12\%) oder auf ein anderes Arbeitszeitkonto übertragen werden (3\%). ${ }^{4}$

\subsection{Regulierung}

Bei Arbeitszeitkonten liegt die Regelungs- und Ausgestaltungskompetenz im Wesentlichen auf der sektoralen bzw. betrieblichen Ebene. Das Arbeitszeitgesetz (ArbZG) beinhaltet dagegen keine gesetzlichen Regelungen zu Arbeitszeitkonten. Lediglich Langzeit- und Lebensarbeitszeitkonten werden durch das Flexi- und Flexi-II-Gesetz im Sozialgesetzbuch, Viertes Buch (SGB IV) geregelt (Seifert, Kümmerling \& Riedmann, 2013).

Nach $\S 1$ des Tarifvertragsgesetzes (TVG) können die Tarifvertragsparteien die Arbeitszeitdauer und -verteilung festlegen. In den letzten zwei Jahrzehnten ist die Tarifbindung aber deutlich zurückgegangen und seit Mitte der 1980er Jahre sind die Kompetenzen zur Arbeitszeitgestaltung von der tariflichen auf die betriebliche Ebene verschoben worden. Diese Kompetenzverschiebung ist auf das Nebeneinander von Arbeitszeitverkürzung und flexibilisierung in den Tarifvereinbarungen der Metallindustrie zurückzuführen.

Nach einem mehrwöchigen Streik im Jahre 1984 verband der sogenannte „Leber-Kompromiss" die Forderung der Gewerkschaft IG Metall nach einer kürzeren Wochenarbeitszeit mit der Forderung der Arbeitgeber nach erweiterten Möglichkeiten zur Arbeitszeitflexibilisierung. Diese Kompromissstruktur prägte alle weiteren Tarifrunden und den

2 Darüber hinaus gibt es vor allem in Tarifbereichen der IG Metall die Möglichkeit einer dauerhaften Verlängerung der regelmäßigen Arbeitszeit. Diese Form der Arbeitszeitverlängerung gilt als eine Sonderform des Arbeitszeitkorridormodells (Bispinck, 2005).

3 Seifert, Kümmerling \& Riedmann (2013) kommen in ihrer Studie zu dem Ergebnis, dass angesammelte Guthaben auf Langzeitkonten in den meisten Betrieben für einen vorzeitigen Ruhestand genutzt wurden. Allerdings gab auch ein Viertel der Betriebe Entnahmen zum Ausgleich von Schwankungen an, obwohl die gesetzlichen Regelungen dies nicht vorsehen.

4 Weitere Befunde dazu finden sich auch bei Bauer, Groß, Munz \& Sayin (2002). 
Betrieben der Metallindustrie wurde tarifvertraglich ein zunehmender Flexibilisierungsspielraum eingeräumt. Die Öffnung der Tarifverträge für flexible Arbeitszeiten erzwang eine Delegation der Aushandlungskompetenzen auf die betriebliche Ebene, wodurch Arbeitszeiten vermehrt dezentral zwischen Betriebsleitung und Betriebs-/Personalräten verhandelt werden. Damit sollte es Betrieben ermöglicht werden, die Arbeitszeiten besser an betriebsspezifische Belange anzupassen (Engfer, 1982; Herrmann, Promberger, Singer \& Trinczek, 1999; Berg, 2008). So überlassen auch die weit verbreiteten tariflichen Regelungen zu Arbeitszeitkonten (Bispinck, 2014) den Betriebsparteien einen weiten Entscheidungsspielraum, um Arbeitszeitkonten an die betriebsspezifische Situation anzupassen. ${ }^{5}$ Weiterhin sieht das Betriebsverfassungsgesetz (BetrVG) vor, dass Betriebsräte bei der Einführung und Überwachung von Arbeitszeitkonten zu beteiligen sind ( $\$ 87$ BetrVG). Insgesamt sind Betriebs-/Personalräte zu einem zunehmend wichtigen Faktor in der betrieblichen Arbeitszeitgestaltung geworden, während Gewerkschaften an Einfluss verloren haben.

\section{Hypothesen}

\subsection{Industrielle Beziehungen}

Bei den Bestimmungsfaktoren von Arbeitszeitkonten werden erstens die industriellen Beziehungen als bedeutend erachtet, welche durch die Tarifbindung und einen existierenden Betriebs-/Personalrat berücksichtigt werden.

Arbeitszeitkonten werden in Tarifverträgen und Betriebsvereinbarungen geregelt (vgl. Kapitel 3.2). Dabei legen Tarifverträge die Rahmenbedingungen fest, die in Betriebsvereinbarungen auf die betriebliche Situation zugeschnitten werden können. Die tarifvertraglichen Regelungen senken für die Betriebsparteien Transaktionskosten, wie Anbahnungs-, Vereinbarungs- oder Abwicklungskosten (Coase, 1960; Picot, 1982; vgl. dazu Dietl 2007). Denn auf betrieblicher Ebene werden Verhandlungen zwischen Arbeitgebern und Arbeitnehmern zu Arbeitszeitkonten erheblich vereinfacht und vermindert. Betriebs-/Personalräte können solche Transaktionskosten weiter senken. Als Interessenvertreter der Beschäftigten verhandeln sie mit Arbeitgebern. Folglich müssen Arbeitgeber nicht mit jedem einzelnen Beschäftigten verhandeln und Beschäftigte müssen sich nicht untereinander verständigen (Ludewig, 2001). Durch die Kompetenzverschiebung von der sektoralen auf die betriebliche Ebene dürften Betriebs-/Personalräte bei der Arbeitszeitgestaltung eine zunehmend wichtigere Stellung einnehmen.

In den Verhandlungen können sich Arbeitnehmervertreter für Arbeitszeitkonten aussprechen, wenn dadurch eine größere Arbeitszeitautonomie für Beschäftigte erwartet wird (Ellguth \& Promberger, 2004). Diese antizipierte Arbeitszeitautonomie hängt im Wesentlichen davon ab, ob und in welchem Maße Beschäftigte Verfügungsrechte über ihre Zeit erhalten und damit über den Auf- und Abbau von Stunden entscheiden können (Lindecke, 2015). Ein weiterer wichtiger Grund für die Befürwortung von Arbeitszeitkonten ist die implizite Versicherung gegen Entlassungsrisiken. Arbeitszeitkonten bieten eine zeitlich be-

5 Einen Überblick über tarifvertragliche Regelungen zu Arbeitszeitkonten bietet Bispinck (2005, 2014). 
fristete Beschäftigungsgarantie und versichern das Risiko, den Arbeitsplatz infolge von negativen Nachfrageveränderungen zu verlieren (Carstensen, 1999).

Gewerkschaften und Betriebs-/Personalräte können sich aber auch gegen Arbeitszeitkonten aussprechen. Zum einen können sie Arbeitszeitkonten bei Wahrnehmung ihrer traditionellen Schutzfunktion eher skeptisch gegenüberstehen, wenn durch die Arbeitszeitflexibilisierung höhere Belastungen für die Beschäftigten erwartet werden oder sich die Arbeitszeiten nur an betrieblichen Bedürfnissen orientieren (Seifert, 1996). Zum anderen werden durch transitorische Überstunden auf Arbeitszeitkonten bezahlte und teils mit Zuschlägen versehene Überstunden verringert oder vermieden, was zu Lohneinbußen bei den Beschäftigten führt. Ebenfalls sind transitorische Überstunden im Vergleich zu bezahlten Überstunden nicht mehr zustimmungspflichtig, wodurch Betriebs-/Personalräte an Verhandlungsmacht einbüßen. Daher ist der Zusammenhang zwischen der Tarifbindung bzw. einem existierenden Betriebs-/Personalrat und Arbeitszeitkonten zunächst unbestimmt und es werden zwei alternative Hypothesen formuliert:

Hla: In tarifgebundenen Betrieben und in Betrieben mit Betriebs-/Personalrat fällt die Wahrscheinlichkeit von Arbeitszeitkonten höher aus als in tarifungebundenen Betrieben und Betrieben ohne Betriebs-/Personalrat.

H1b: In tarifgebundenen Betrieben und in Betrieben mit Betriebs-/Personalrat fällt die Wahrscheinlichkeit von Arbeitszeitkonten geringer aus als in tarifungebundenen Betrieben und Betrieben ohne Betriebs-/Personalrat.

\subsection{Instrumente externer Flexibilität}

Zweitens wird der Einsatz von externen Flexibilitätsinstrumenten als ein weiterer Bestimmungsfaktor für Arbeitszeitkonten in Betrieben erachtet. Dazu zählen unter anderem Neueinstellungen, Entlassungen, Befristungen und Leiharbeit, die von den Betrieben (bis zu einem gewissen Grad) substitutiv oder komplementär zu internen Instrumenten eingesetzt werden können.

Neueinstellungen sind für Betriebe eine unsichere Investitionsentscheidung, denn zum Zeitpunkt der Einstellung haben sie keine vollständigen Informationen über die Leistungsfähigkeit von Beschäftigten (Spence, 1973). Auch entstehen betriebliche Such-, Auswahl- und Einarbeitungskosten (Bellmann \& Alda, 2004). Betriebe sind daher interessiert Neueinstellungen (möglichst lange) zu vermeiden. Dies ist insbesondere dann der Fall, wenn es sich um kurzfristige, saisonale oder zyklische Schwankungen handelt, die nur eine vorübergehende Anpassung des Arbeitseinsatzes an das Arbeitsaufkommen erfordern. In diesem Fall nutzen Betriebe Arbeitszeitkonten substitutiv zu Neueinstellungen, um auf diesen Umwelteinfluss zu reagieren. Eine Anpassung des Arbeitseinsatzes durch Arbeitszeitkonten stößt jedoch auch an Grenzen. Bei einer langfristig steigenden Nachfrage wird eine dauerhafte Arbeitszeitverlängerung durch gesetzliche, tarifvertragliche und/oder betriebliche Vereinbarungen begrenzt und Neueinstellungen werden notwendig. In diesem Fall werden Arbeitszeitkonten komplementär zu Neueinstellungen genutzt. Im Zuge von Personalrekrutierungen können Betriebe Arbeitszeitkonten auch als Marketinginstrument nutzen (Goudswaard et al., 2012) und so ihre Attraktivität als Arbeitgeber steigern. Diese intendierte Steigerung der Arbeitgeberattraktivität stellt insbesondere in den letzten Jahren einen 
immer wichtiger werdenden Faktor dar, da geeignete Arbeitskräfte in einigen Berufen zunehmend knapper werden. Bei Neueinstellungen und Arbeitszeitkonten wird sowohl von einem negativen als auch einem positiven Zusammenhang ausgegangen und es werden zwei alternative Hypothesen formuliert:

H2a: Mit einem zunehmenden Anteil an Neueinstellungen sinkt die Wahrscheinlichkeit von Arbeitszeitkonten in Betrieben.

H2b: Mit einem zunehmenden Anteil an Neueinstellungen steigt die Wahrscheinlichkeit von Arbeitszeitkonten in Betrieben.

Entlassungen ziehen arbeits- und sozialrechtlich bedingte Kosten, wie Abfindungszahlungen, nach sich und betriebliches Humankapital geht verloren (Carstensen, 1999; Seifert, 2003). Aufgrund eigener Humankapitalinvestitionen sind Betriebe aber daran interessiert das angesammelte Humankapital der Beschäftigten im Betrieb zu halten und effizient zu nutzen. Bei vorübergehenden negativen Nachfrageschwankungen werden Entlassungen deshalb möglichst vermieden (Erlinghagen, 2005) und zunächst angesammelte Guthaben auf Arbeitszeitkonten abgebaut, um die Arbeitszeit vorübergehend zu verringern. Bei langfristig negativen Nachfrageveränderungen können jedoch Entlassungen notwendig werden, sofern andere Möglichkeiten zur Variation des Arbeitseinsatzes ausgeschöpft wurden. Dabei begrenzt der in Deutschland vergleichsweise hohe Kündigungsschutz die betrieblichen Möglichkeiten von Entlassungen. Bei Entlassungen und Arbeitszeitkonten kann deshalb ebenfalls von einem negativen und positiven Zusammenhang ausgegangen werden:

H3a: Mit einem zunehmenden Anteil an Entlassungen sinkt die Wahrscheinlichkeit von Arbeitszeitkonten in Betrieben.

H3b: Mit einem zunehmenden Anteil an Entlassungen steigt die Wahrscheinlichkeit von Arbeitszeitkonten in Betrieben.

Befristungen bieten für Betriebe Kostenvorteile, da sie zum Vertragsende auslaufen können und keine Kündigungs- und Entlassungskosten nach sich ziehen. Befristungen stellen vor allem dann eine Alternative zu internen Instrumenten, wie Arbeitszeitkonten, dar, wenn kein unternehmensspezifisches Humankapital der Beschäftigten benötigt wird und deshalb ein leichterer Beschäftigtenaustausch erfolgen kann. Befristungen werden aber auch komplementär zu internen Flexibilitätsinstrumenten genutzt. Dies dürfte vor allem für Betriebe mit einer stabilen Kern- oder Stammbelegschaft gelten. Denn eine stabile Kern- oder Stammbelegschaft mit unbefristeten Arbeitsverträgen begrenzt die betrieblichen Reaktionsmöglichkeiten bei Nachfrageschwankungen auf vor allem interne Flexibilität. Um die betrieblichen Reaktionsmöglichkeiten zu vergrößern und sowohl intern als auch extern flexibel zu sein, werden Beschäftigte befristet eingestellt. Damit bauen Betriebe parallel zur Kern- oder Stammbelegschaft eine Randbelegschaft auf. Betriebe greifen auch auf Befristungen zurück, um Arbeitskräfte zunächst länger zu erproben und diese bei Eignung in ein unbefristetes Beschäftigungsverhältnis zu übernehmen. (Giesecke, 2006; Hohendanner \& Ramos Lobato, 2017). In den letzten Jahren ist der Anteil der befristeten Beschäftigungsverhältnisse an der betrieblichen Gesamtbeschäftigung von rund 6,2 auf 8,2 Prozent gestiegen (Hohendanner, Ostmeier \& Ramos Lobato, 2015), womit Befristungen bei den betrieb- 
lichen Bestimmungsfaktoren von Arbeitszeitkonten eine zunehmend wichtigere Rolle spielen könnte. Durch den unbestimmten Zusammenhang werden zwei alternative Hypothesen formuliert:

H4a: Mit einem zunehmenden Anteil befristet Beschäftigter sinkt die Wahrscheinlichkeit von Arbeitszeitkonten in Betrieben.

H4b: Mit einem zunehmenden Anteil befristet Beschäftigter steigt die Wahrscheinlichkeit von Arbeitszeitkonten in Betrieben.

Auch Leiharbeitsverhältnisse haben in den letzten Jahren an Bedeutung gewonnen. Der durchschnittliche Bestand an Leiharbeitsverhältnissen ist von rund 328.000 im Jahr 2000 auf rund $878.000 \mathrm{im}$ Jahr 2012 gestiegen. Leiharbeit ermöglicht eine schnelle Anpassung bei einem unvorhergesehenen Personalbedarf. Dabei werden (lange) Suchprozesse und Transaktionskosten, wie Such-, Anbahnungs- und Vereinbarungskosten zwischen Arbeitgebern und Arbeitnehmern vermieden, auch entfallen spätere Entlassungskosten. Vor diesem Hintergrund können Betriebe Leiharbeit alternativ zu Arbeitszeitkonten einsetzen. Gegen diese Substitution sprechen im Wesentlichen aber zwei Gründe: Erstens ist die Leiharbeit aufgrund des spezifischen Verhältnisses zwischen Verleihbetrieb, Entleihbetrieb und Leiharbeitnehmer für die Entleihbetriebe relativ teuer. Daher ist davon auszugehen, dass Betriebe die Leiharbeit komplementär zu anderen internen Instrumenten einsetzen, um auf allen Ebenen flexibel agieren zu können. Dies dürfte vor allem für Betriebe mit dynamischen, komplexen und unsicheren Unternehmensumwelten gelten, womit ein hohes Maß interner und externer Flexibilität erforderlich ist. Zweitens übernimmt die Leiharbeit eine Pufferfunktion für die Stammbelegschaft. Betriebe möchten Stammbelegschaften mit ihrem spezifischen Humankapital halten. Bei negativen Nachfrageveränderungen wird deshalb zunächst die Anzahl der Leiharbeitskräfte verringert, bevor Stammbeschäftigte entlassen werden. Aus diesem Grund ging die Zahl der Leiharbeitnehmer während der Finanz- und Wirtschaftskrise 2008/2009 deutlich zurück (Haller \& Jahn, 2014). Der Zusammenhang zwischen Leiharbeit und Arbeitszeitkonten ist ebenfalls unbestimmt, wodurch zwei alternative Hypothesen formuliert werden:

H5a: Mit einem zunehmenden Anteil an Leiharbeitern sinkt die Wahrscheinlichkeit von Arbeitszeitkonten in Betrieben.

H5b: Mit einem zunehmenden Anteil an Leiharbeitern steigt die Wahrscheinlichkeit von Arbeitszeitkonten in Betrieben.

\subsection{Vertraglich vereinbarter Arbeitszeitumfang}

Drittens ist der vertraglich vereinbarte Arbeitszeitumfang bedeutend, der die betriebliche Beschäftigungsstruktur kennzeichnet. Neben einer Vollzeitstelle können Arbeitnehmer auch in Teilzeit- oder geringfügiger Beschäftigung arbeiten, wobei letztere eine Sonderform der Teilzeitbeschäftigung ist.

Ein geringfügiges Beschäftigungsverhältnis ist für Arbeitgeber zunächst teurer als eine sozialversicherungspflichtige Beschäftigung. Denn bei geringfügigen Beschäftigungsverhältnissen belaufen sich die Abgaben der Arbeitgeber auf rund 30 Prozent, während diese 
bei sozialversicherungspflichtigen Beschäftigungen bei rund 21 Prozent liegen. Arbeitgeber kompensieren diese höhere Abgabenquote allerdings durch erheblich niedrigere Stundenlöhne von geringfügig Beschäftigten (Voss \& Weinkopf, 2012). Betriebe nutzen geringfügige Beschäftigungen, um den Arbeitseinsatz flexibel an vorübergehende Auftragsschwankungen anzupassen. Dies erscheint vor allem dann nachvollziehbar, wenn die Schwankungen zu gering sind, um eine neue Vollzeitstelle zu schaffen. Weiterhin werden geringfügig Beschäftigte aufgrund ihrer geringeren Stundenzahl flexibler als Beschäftigte in einem Normalarbeitsverhältnis eingesetzt. Betriebe decken damit einen zusätzlichen Personalbedarf am Wochenende oder außerhalb der Kernarbeitszeiten ab. Dies dürfte vor allem auf verschiedene Dienstleistungsbranchen, wie den Handel oder das Gastgewerbe, zutreffen, wo die geringfügige Beschäftigung verbreitet ist (Bäcker, 2006).

Für Teilzeitbeschäftigungen spricht aus betrieblicher Sicht die Möglichkeit, Betriebs- und Arbeitsabläufe flexibel zu gestalten. Auch lassen sich vorübergehende Personalengpässe eher überbrücken (Wanger, 2011). Im Zuge der Variation des Arbeitseinsatzes durch geringfügige Beschäftigung und Teilzeitarbeit kann auch ein dauerhafter Einsatz von (transitorischen) Überstunden vermieden werden, der Beschäftigte langfristig belasten kann. Da die Anzahl der Männer und Frauen in einer geringfügigen und Teilzeitbeschäftigung gestiegen ist (Wanger, 2011), könnten diese Beschäftigungsformen im Zeitverlauf eine zunehmend wichtigere Rolle in Bezug auf Arbeitszeitkonten spielen. Dabei wird folgende Hypothese formuliert:

H6: Mit einem steigenden Anteil von geringfügig- und Teilzeitbeschäftigten fällt die Wahrscheinlichkeit von Arbeitszeitkonten in Betrieben geringer aus.

\subsection{Individuelle Merkmale der Beschäftigten}

Viertens sind individuelle Faktoren der Beschäftigten, wie das Geschlecht und die Qualifikation, bedeutend, die ebenfalls die betriebliche Beschäftigungsstruktur bestimmen.

Die Geschlechterverteilung wird durch den Frauenanteil an der Gesamtbelegschaft gemessen. Frauen müssen häufig Erwerbs-, Familien- und Hausarbeit vereinbaren und erfahren hohe Belastungen. Auch sind sie häufiger von Einschränkungen in den zeitlichen Verfügungsmöglichkeiten betroffen, in deren Folge sie häufiger Zeitdruck und Zeitnot verspüren. Dadurch ist ihre Zeit beschränkt, länger zu arbeiten und (langfristig) Guthaben auf Arbeitszeitkonten aufzubauen (Wotschack, 2012). Anstelle einer Variation von Arbeitszeitdauer und -lage über mehrere Wochen oder Monate benötigen Frauen ,alltägliche“ Flexibilität, das heißt eine Möglichkeit die tägliche Arbeitszeitlänge und -lage zu variieren, um Beruf und Familie zu vereinbaren. Jedoch widerspricht eine Arbeitszeitvariation nach individuellen Bedürfnissen dem betrieblichen Interesse einer kontinuierlichen Verfügbarkeit von Arbeitskräften (Promberger, 2005). Da die Zahl der beschäftigten Frauen seit den 1990er Jahren deutlich zugenommen hat, dürfte es für Betriebe schwieriger sein, Arbeitszeitkonten für betriebliche Bedürfnisse zu nutzen. Dies spricht für folgende Hypothese:

H7: Mit einem zunehmenden Anteil an Frauen fällt die Wahrscheinlichkeit von Arbeitszeitkonten in Betrieben geringer aus.

Das betriebliche Qualifikationsniveau wird durch den Anteil der qualifizierten Beschäftigten gemessen. Qualifizierte Arbeitskräfte können eine kritische Ressource sein (Pfeffer \& 
Salancik, 1978), die Betriebe extern auf dem Arbeitsmarkt beziehen müssen. Dabei entwickelt sich zwischen den Betrieben ein zunehmender Kampf um qualifizierte Arbeitskräfte und in einigen Berufen treten bereits Engpässe auf. Beschäftigte stellen für die Überlassung ihrer Ressource „Arbeitskraft“ Ansprüche an den Betrieb, die mit zunehmendem Qualifikationsniveau steigen. Forderungen können beispielsweise Arbeitsplatzsicherheit, pünktliche Lohn-/Gehaltszahlungen und gute Arbeitsbedingungen sein (Pfeffer \& Salancik, 1978; Pearce, 1982; Sheppard, 1985; vgl. dazu auch Brunner 2009). Betriebe können diesen Forderungen begegnen und Arbeitszeitkonten einführen, um die Arbeitsplatzsicherheit zu erhöhen und Arbeitsbedingungen zu verbessern. Ebenfalls investieren Betriebe in die Weiterbildung von qualifizierten Beschäftigten, um das betriebsspezifische Humankapital weiter zu verbessern. Das arbeitgeberseitige Interesse an langfristigen Arbeitsverhältnissen steigt, da betriebliche Investitionskosten zu amortisieren sind (Hinrichs, 1992; Bundesmann-Jansen, Groß \& Munz, 2000). Vor diesem Hintergrund werden Betriebe versuchen externe Flexibilität (möglichst lange) zu vermeiden (Erlinghagen, 2005). Stattdessen können sie interne Instrumente nutzen, um die Beschäftigung zu stabilisieren. Daher kann von folgendem $\mathrm{Zu}$ sammenhang ausgegangen werden:

H8: Mit einem steigenden Anteil qualifizierter Beschäftigter steigt die Wahrscheinlichkeit von Arbeitszeitkonten in Betrieben.

\section{Daten, Variablen und Methode}

\subsection{IAB-Betriebspanel}

Für die empirischen Analysen werden die Daten des IAB-Betriebspanels genutzt. Das I$\mathrm{AB}-$ Betriebspanel ist eine repräsentative Arbeitgeberbefragung $\mathrm{zu}$ den betrieblichen $\mathrm{Be}-$ stimmungsgrößen der Arbeitsnachfrage und bietet unter anderem Informationen zu Beschäftigung, zur Lohnhöhe und zu Arbeits- und Betriebszeiten. Jährlich werden rund 16.000 Betriebe aller Branchen und Betriebsgrößen durch TNS Infratest Sozialforschung $\mathrm{GmbH}$ befragt. Die Grundgesamtheit bilden alle Betriebe mit mindestens einem sozialversicherungspflichtig Beschäftigten in Deutschland (Bellmann, 2002; Fischer, Janik Müller, \& Schmucker, 2009).

\subsection{Verwendete Variablen}

Informationen zu Arbeitszeitkonten stehen in den Jahren 1999, 2002, 2004, 2006 und von 2008 bis 2012 zur Verfügung. Die entsprechende Frage lautet: „Gibt es in Ihrem Betrieb/ Ihrer Dienststelle Regelungen zu Arbeitszeitkonten, also von der Gleitzeit bis hin zu Jahresarbeitszeitvereinbarungen? Oder sind solche Regelungen geplant?"6 Dies bildet die Grundlage für die abhängige Variable Arbeitszeitkonten im Betrieb, die als Dummyvariable $(0 / 1)$ kodiert wird. Sie nimmt den Wert 1 an, wenn Regelungen zu Arbeitszeitkonten im

6 Diese Frage erfasst die betriebliche Verbreitung von Kurzzeitkonten, Informationen zu Langzeitkonten werden in einer separaten Frage erhoben. In den nachfolgenden Analysen stehen ausschließlich die Kurzzeitkonten im Mittelpunkt der Betrachtung. 
Betrieb vorhanden sind; sie nimmt den Wert 0 an, wenn Regelungen geplant oder weder vorhanden noch geplant sind. Sofern Arbeitszeitkontenregelungen vorhanden sind, wird gefragt für wie viel Prozent der Beschäftigten diese vorhandenen Regelungen gelten. Diese Frage ist Grundlage für die abhängige Variable Partizipationsrate der Beschäftigten, d. h. die Verbreitungsquote von Arbeitszeitkonten bei Beschäftigten in Betrieben mit Arbeitszeitkonten. Die Variable ist metrisch und liegt zwischen 0 und 100 Prozent.

Bei den unabhängigen Variablen sind im Kontext der industriellen Beziehungen die Tarifbindung und ein existierender Betriebs- oder Personalrat relevant. Beide Variablen sind als Dummyvariablen (0/1) kodiert. Die Tarifbindung erhält den Wert 1, wenn ein Branchentarifvertrag oder ein Haus- oder Firmentarifvertrag im Betrieb existiert; sie erhält den Wert 0, wenn kein Tarifvertrag existiert. Die Variable Betriebs-/Personalrat (0/1) unterscheidet zwischen Betrieben mit einem Betriebs-oder Personalrat und Betrieben ohne Betriebs- oder Personalrat. Zur zweiten Gruppe der Instrumente externer Flexibilität zählen die Anzahl der Neueinstellungen und Kündigungen durch den Betrieb im 1. Halbjahr des Befragungsjahres, die in Relation zur Beschäftigtenzahl gesetzt werden. Der Anteil der befristet Beschäftigten und der Leiharbeiter werden jeweils als Anteil an der Gesamtbelegschaft berücksichtigt. Die dritte Gruppe bezieht sich auf den vertraglich vereinbarten Arbeitszeitumfang. Dazu zählen der Anteil der Teilzeitbeschäftigten und der geringfügig Beschäftigten. Die vierte Gruppe beschreibt individuelle Merkmale der Beschäftigten und umfasst den Frauenanteil und den Anteil der Facharbeiter und Angestellten/Beamten mit qualifizierter Tätigkeit.

Neben diesen interessierenden Variablen werden Kontrollvariablen berücksichtigt, für die ebenfalls ein Zusammenhang mit Arbeitszeitkonten vermutet werden kann. Dazu wird erstens die logarithmierte Betriebsgröße in das Modell aufgenommen. Zweitens umfasst die Variable Wirtschaftsbereich 14 verschiedene Branchen, Referenzkategorie ist das Baugewerbe. Drittens differenziert die Art des Betriebs/der Dienststelle zwischen vier verschiedenen Arten, Referenzkategorie ist ein eigenständiges Unternehmen oder eine eigenständige Einrichtung. Viertens wird der technische Stand der Anlagen und der Betriebs- und Geschäftsausstattung des befragten Betriebs im Vergleich zu anderen Betrieben in der Branche berücksichtigt. Die fünfstufige Skala von (1) auf dem neuesten Stand bis (5) völlig veraltet wurde zu den drei Ausprägungen neue Technologie, alte Technologie und weder neue noch alte Technologie (Referenzkategorie) gruppiert. Fünftens beschreibt die Variable Investitionen in EDV, Informations- und Kommunikationstechnik, ob im vergangenen Geschäftsjahr Investitionen in diesem Bereich getätigt wurden. Sechstens wird zwischen Betrieben in West- und Ostdeutschland differenziert.

Siebtens wird für weitere Instrumente externer Flexibilität kontrolliert, um diese hinreichend abzubilden. Darunter fallen der Anteil nicht übernommener Beschäftigter, d. h. der Anteil an ausgeschiedenen Beschäftigten nach Abschluss einer betrieblichen Ausbildung, der Anteil von Beschäftigten mit auslaufendem Arbeitsvertrag, der Anteil von Beschäftigten mit einvernehmlicher Aufhebung, der Anteil von ausgeschiedenen Beschäftigten in den Ruhestand, d. h. Beschäftigte, die nach der regulären Altersgrenze in den Ruhestand eingetreten sind und der Anteil von ausgeschiedenen Beschäftigten in die Frührente. ${ }^{7}$

7 Da im Gegensatz zu Neueinstellungen und Entlassungen der Flexibilitätsspielraum durch diese Instrumente nicht ausschließlich von betrieblicher Seite beeinflussbar ist, wird hier auf eine detailliertere Darstellung verzichtet. 


\subsection{Methode}

Für die Analysen wurden logistische Regressionsmodelle verwendet, womit die Wahrscheinlichkeit modelliert wird, dass ein bestimmtes Ereignis, wie Arbeitszeitkonten in einem Betrieb, eintritt und zwar unter dem Einfluss der unabhängigen Variablen (vgl. z.B. Greene, 2008). In der logistischen Regression können Koeffizienten ausgewiesen werden. Diese sind inhaltlich aber kaum sinnvoll zu interpretieren, sie ermöglichen jedoch eine Angabe der Richtung des Zusammenhangs. Deshalb werden in den Schätzungen die durchschnittlichen marginalen Effekte (average marginal effects, AME) ausgewiesen. Sie sind leichter interpretierbar und zeigen, um wie viele Prozentpunkte sich die Wahrscheinlichkeit von Arbeitszeitkonten in einem Betrieb im Mittel aller Beobachtungen verändert, wenn die unabhängige Variable um eine Einheit steigt (Auspurg \& Hinz, 2011). ${ }^{8}$ Die logistischen Regressionen werden um OLS-Schätzungen ergänzt und es wird analysiert, von welchen Faktoren die Verbreitungsquote von Arbeitszeitkonten bei Beschäftigten in Betrieben mit Arbeitszeitkonten abhängt. Die AME zeigen, um wie viele Prozentpunkte sich die Verbreitungsquote ändert, wenn die unabhängige Variable um eine Einheit steigt. In OLS-Regressionen entsprechen die marginalen Effekte den Koeffizienten.

Die Schätzungen wurden jeweils getrennt als Querschnitt und gepoolt für die Zeitpunkte 2006, 2009 und 2012 durchgeführt. Dabei standen die interessierenden Merkmale zu allen drei Zeitpunkten zur Verfügung. Ein Vergleich einzelner Zeitpunkte gibt Aufschluss darüber, ob es Unterschiede in der Wirkung von Merkmalen über die Zeit gibt, das heißt, ob die Effekte in einzelnen Jahren stärker oder schwächer bzw. signifikant oder insignifikant werden. Starke Unterschiede weisen auf eine nicht zeitkonstante unbeobachtete Heterogenität hin. Mit dem Wald Test wurde geprüft, ob Unterschiede über die Zeit statistisch signifikant sind. Die Nullhypothese besagt, dass es keine signifikanten Unterschiede gibt. ${ }^{9}$

Die Jahre 2006 und 2012 sind durch konjunkturelle Aufschwungphasen gekennzeichnet, während das Jahr 2009 mit der Finanz- und Wirtschaftskrise eine konjunkturelle Abschwungphase ist. Vor dem Hintergrund der großen Wucht der Finanz- und Wirtschaftskrise und der intensiven Nutzung von Arbeitszeitkonten zur Abfederung von negativen Beschäftigungsfolgen ist es von besonderem Interesse, ob sich im Jahr 2009 zusätzliche oder andere Determinanten von Arbeitszeitkonten zeigen. Mit dem gewählten Vorgehen lassen sich zeitliche Entwicklungen bei den verschiedenen Determinanten von Arbeitszeitkonten adäquat darstellen, die im Rahmen von Panelanalysen nicht geleistet werden können.

8 Bei Dummyvariablen ist die Terminologie „marginaler Effekt“ streng genommen nicht richtig, da es sich um die (ceteris paribus) mittlere Differenz der abhängigen Variable zwischen zwei Gruppen handelt (vgl. z.B. Buis, 2010). Zur leichteren Lesbarkeit des Textes wird der Begriff dennoch auch bei Dummyvariablen verwendet.

9 Auspurg und Hinz (2011) führen am Beispiel der Bildungschancen im Kohortenvergleich einen Chi²-Wert Test durch und prüfen damit, ob Unterschiede in den marginalen Effekten der Kohorten statistisch signifikant sind. 


\section{Arbeitszeitkonten in der betrieblichen Praxis}

\subsection{Verbreitung nach Betriebsgröße}

Insgesamt ist der Anteil der Betriebe bzw. Dienststellen mit Regelungen zu Arbeitszeitkonten gestiegen. Während im Jahr 2006 rund 21 Prozent der Betriebe mit mindestens einem sozialversicherungspflichtig Beschäftigten angaben, über Arbeitszeitkonten zu verfügen, waren es im Jahr 2012 rund 28 Prozent (s. Tabelle 1).

Im Vergleich zu anderen europäischen Staaten sind Arbeitszeitkonten in Deutschland weit verbreitet. So zeigen die Ergebnisse der europäischen Unternehmenserhebung 2009, dass in den EU-27 Ländern durchschnittlich rund 38 Prozent der Betriebe mit mindestens zehn Beschäftigten Arbeitszeitkonten haben. In Deutschland liegt die Verbreitung in diesen Betrieben bei rund 50 Prozent. Am weitesten verbreitet sind Arbeitszeitkonten in den nordeuropäischen Ländern, wie in Finnland (über 70 Prozent) oder in Dänemark und Schweden (über 50 Prozent), während in Bulgarien und Griechenland vergleichsweise wenige Betriebe Arbeitszeitkonten führen (rund 20 bzw. 10 Prozent) (Riedmann, Van Gyes, Román, Kerkhofs \& Bechmann, 2010).

Arbeitszeitkonten kommen in größeren Betrieben deutlich häufiger vor als in kleineren Betrieben. Grund für die unterschiedliche Verbreitung ist der verhältnismäßig große Aufwand, der mit einer Arbeitszeitkonteneinführung einhergeht. Dieser Aufwand übersteigt in kleineren Betrieben den Nutzen einer formalen Flexibilisierung. Eine flexible Arbeitszeitgestaltung scheint hier eher informell geregelt zu sein, womit sich Arbeitszeitkonten kaum lohnen (Bosch, 1996; Bellmann \& Gewiese, 2003). Dagegen ist der hohe Anteil der Arbeitszeitkontenbetriebe unter den Großbetrieben auch auf den Fixkostencharakter von Arbeitszeitkonten zurückzuführen. Bei der Einführung fallen Transaktionskosten (z.B. Anbahnungs-, Vereinbarungskosten) sowie Kosten für Pflege und Dokumentation an. Aufgrund der Fixkostendegression sinken die durchschnittlichen Kosten von Arbeitszeitkonten mit zunehmender Betriebsgröße, wodurch dieses Instrument für größere Betriebe günstiger wird (Ludewig, 2001).

Bei einer detaillierteren Betrachtung der Verbreitung von Arbeitszeitkonten nach der Betriebsgröße zeigt sich eine unterschiedliche Dynamik bei Zunahme und Struktur. Arbeitszeitkonten sind in Kleinst- und Kleinbetrieben zwar immer noch im geringsten Maße verbreitet, in Relation zum Jahr 2006 steigt der Anteil in diesen Betrieben jedoch am stärksten. In Großbetrieben steigt der Anteilrelativ betrachtet am wenigsten. Der Gesamtanstieg des Anteils der Arbeitszeitkontenbetriebe um rund 34 Prozent wird somit vor allem von den Kleinst- und Kleinbetrieben getragen.

Diese Entwicklung wird auch bei einem Vergleich der Anteile der Arbeitszeitkontenbetriebe nach der Größe an allen Arbeitszeitkontenbetrieben und der Anteile der Betriebe nach der Größe über alle Betriebe bestätigt. Bei größeren Betrieben sind die Anteile an allen Arbeitszeitkontenbetrieben absolut und relativ betrachtet zurückgegangen. Bei den Kleinstbetrieben zeigt sich zwar ebenfalls ein leichter Rückgang, allerdings ist dieser vergleichsweise gering. Dagegen steigt in der Gruppe der Kleinbetriebe der Anteil mit Arbeitszeitkonten an allen Arbeitszeitkontenbetrieben. 
Tabelle 1: Verbreitung und Struktur von (Arbeitszeitkonten-)Betrieben nach Betriebsgröße (Anteil der Betriebe und Veränderungsrate in Prozent)

\begin{tabular}{lcrr}
\hline $\begin{array}{l}\text { Anteil der Betriebe mit Arbeitszeitkonten nach Größenklasse } \\
\text { an allen Betrieben }\end{array}$ & $\mathbf{2 0 0 6}$ & $\mathbf{2 0 1 2}$ & $\begin{array}{c}\text { Veränderung in \% } \\
\text { (2012 gegenüber 2006) }\end{array}$ \\
\hline 1-9 Beschäftigte & 14,6 & 19,3 & 32,2 \\
10-49 Beschäftigte & 32,7 & 43,5 & 33,0 \\
50-249 Beschäftigte & 60,6 & 66,1 & 9,1 \\
250 und mehr Beschäftigte & 74,9 & 79,6 & 6,3 \\
Gesamt & 20,9 & 28,0 & 33,9 \\
\hline
\end{tabular}

Anteil der Betriebe mit Arbeitszeitkonten nach Größenklasse an allen Arbeitszeitkontenbetrieben

\begin{tabular}{|c|c|c|c|}
\hline 1-9 Beschäftigte & 49,2 & 48,1 & $-2,2$ \\
\hline 10-49 Beschäftigte & 35,7 & 38,8 & 8,5 \\
\hline 50-249 Beschäftigte & 12,5 & 10,8 & $-12,9$ \\
\hline 250 und mehr Beschäftigte & 2,6 & 2,3 & $-13,7$ \\
\hline Gesamt & 100,0 & 100,0 & 0,0 \\
\hline \multicolumn{4}{|c|}{ Anteil der Betriebe nach Größenklasse an allen Betrieben } \\
\hline 1-9 Beschäftigte & 72,5 & 69,7 & $-3,9$ \\
\hline 10-49 Beschäftigte & 22,4 & 24,9 & 11,1 \\
\hline 50-249 Beschäftigte & 4,3 & 4,6 & 6,6 \\
\hline 250 und mehr Beschäftigte & 0,7 & 0,8 & 7,5 \\
\hline Gesamt & 100,0 & 100,0 & 0,0 \\
\hline
\end{tabular}

Quelle: IAB-Betriebspanel, hochgerechnete Ergebnisse; eigene Berechnungen.

\subsection{Verbreitungsquote bei Beschäftigten}

Bei der Einführung von Arbeitszeitkonten legen Betriebe fest, ob diese allen oder nur einem Teil der Beschäftigten zur Verfügung gestellt werden. Die Verbreitungsquote beschreibt, wie viel Prozent der Beschäftigten ein Arbeitszeitkonto haben, gegeben dass es diese in den Betrieben bzw. Dienststellen gibt (vgl. Kapitel 5.2). Die Verbreitungsquote ist in allen Größenklassen und Branchen hoch, insgesamt ist der Anteil der Beschäftigten mit Arbeitszeitkonto in einem Arbeitszeitkontenbetrieb von rund 85 auf 88 Prozent gestiegen (s. Tabelle 2). In kleineren Betrieben verfügen anteilig noch mehr Beschäftigte über ein Arbeitszeitkonto, d. h. haben dazu Zugang, als in größeren Betrieben. Eine höhere Verbreitungsquote in kleineren Betrieben erscheint jedoch plausibel, da eine Einführung bei kleineren Betrieben in Relation zur Beschäftigtenzahl höhere Kosten erzeugt. Sofern Arbeitszeitkonten einmal eingeführt sind, steigen die Kosten aber nicht mehr so stark mit der Betriebsgröße. ${ }^{10}$

Bei einer Differenzierung nach Branchen zeigt sich, dass im Jahr 2012 die Verbreitungsquote bei Beschäftigten in der Produktionsgüterindustrie/Grundstoffverarbeitung und im Gastgewerbe am geringsten ist. Die geringere Verbreitungsquote könnte bei ersterer

10 Die höhere Verbreitungsquote in kleineren Betrieben kann auch auf ein statistisches Artefakt zurückgeführt werden. Da die Anzahl der Beschäftigten mit einem Arbeitszeitkonto in Relation zur Gesamtgröße des Betriebs steht, ist in kleineren Betrieben eine höhere Quote auch dann zu erreichen, wenn die Anzahl der Beschäftigten mit einem Arbeitszeitkonto gering ist. Werden beispielsweise in einem Betrieb mit zehn Beschäftigten für fünf der Mitarbeiter Arbeitszeitkonten eingeführt, liegt die Verbreitungsquote bei 50 Prozent, während diese in einem Betrieb mit 20 Beschäftigten nur bei 25 Prozent liegt. 
Branche durch einen höheren Anteil an Leiharbeitern und bei letzterer Branche durch einen höheren Anteil an Saisonkräften und geringfügig Beschäftigten erklärt werden, denen kein Arbeitszeitkonto zur Verfügung gestellt wird. Überdurchschnittlich hoch war die Verbreitungsquote im Jahr 2012 im Gesundheits-, Veterinär- und Sozialwesen sowie im Bereich Kredit und Versicherungen.

Tabelle 2: Verbreitung von Arbeitszeitkonten und Verbreitungsquote bei Beschäftigten in Arbeitszeitkontenbetrieben nach Größe und Branche (Anteil in Prozent)

\begin{tabular}{|c|c|c|c|c|}
\hline & \multicolumn{2}{|c|}{$\begin{array}{l}\text { Anteil der Betriebe } \\
\text { mit Arbeitszeitkonten }\end{array}$} & \multicolumn{2}{|c|}{ Verbreitungsquote } \\
\hline & 2006 & 2012 & 2006 & 2012 \\
\hline \multicolumn{5}{|l|}{ Betriebsgröße } \\
\hline 1-9 Beschäftigte & 14,6 & 19,3 & 88,2 & 90,1 \\
\hline 10-49 Beschäftigte & 32,7 & 43,5 & 82,5 & 87,7 \\
\hline 50-249 Beschäftigte & 60,6 & 66,1 & 81,7 & 84,9 \\
\hline 250 und mehr Beschäftigte & 74,9 & 79,6 & 81,7 & 84,4 \\
\hline \multicolumn{5}{|l|}{ Branche } \\
\hline Land- und Forstwirstschaft/Fischerei & 16,9 & 27,7 & 82,3 & 88,1 \\
\hline Bergbau/Energie- und Wasserversorgung & 59,4 & 53,4 & 89,3 & 88,5 \\
\hline Verbrauchsgüterindustrie/Nahrungs- und Genussmittel & 22,8 & 28,9 & 83,4 & 83,9 \\
\hline Produktionsgüterindustrie/Grundstoffverarbeitung & 40,9 & 44,6 & 81,8 & 83,0 \\
\hline Investitions- und Gebrauchsgüterindustrie & 34,6 & 42,2 & 86,5 & 89,0 \\
\hline Baugewerbe & 28,0 & 32,0 & 82,8 & 87,3 \\
\hline Handel/Reparatur & 17,9 & 24,7 & 86,7 & 87,3 \\
\hline Verkehr/Information und Kommunikation & 18,9 & 27,0 & 84,2 & 90,6 \\
\hline KreditNersicherungen & 20,1 & 24,4 & 87,6 & 91,2 \\
\hline Gastgewerbe & 10,3 & 16,4 & 88,0 & 82,7 \\
\hline Erziehung und Unterricht & 20,8 & 30,7 & 74,6 & 87,4 \\
\hline Gesundheits-/Sozial- und Veterinärwesen & 20,0 & 28,5 & 84,7 & 91,3 \\
\hline Sonstige Dienstleistungen & 17,4 & 25,5 & 86,5 & 89,6 \\
\hline $\begin{array}{l}\text { Organis. ohne Erwerbscharakter/Öffentliche Verwal- } \\
\text { tung/Sozialversicherung }\end{array}$ & 32,7 & 41,2 & 87,0 & 89,7 \\
\hline Gesamt & 20,9 & 28,0 & 85,1 & 88,5 \\
\hline
\end{tabular}

Quelle: IAB-Betriebspanel, hochgerechnete Ergebnisse; eigene Berechnungen.

\section{Ergebnisse aus multivariaten Analysen}

\section{Bestimmungsfaktoren der Verbreitung von Arbeitszeitkonten}

Tabelle 3 stellt die Effekte der verschiedenen betrieblichen Merkmale auf die Wahrscheinlichkeit von Arbeitszeitkonten in einem Betrieb dar. Bei allen Regressionsmodellen wurden die unabhängigen Variablen auf Multikollinearität getestet. Weder die Toleranzindizes und die Varianzinflationsfaktoren der unabhängigen Variablen noch der Konditionsindex weisen auf ein Problem mit Multikollinearität hin.

Tarifgebundene und Betriebe mit Betriebs- oder Personalrat haben im Vergleich zu nicht tarifgebundenen und Betrieben ohne Betriebs- oder Personalrat überdurchschnittlich häufig Arbeitszeitkonten. Dies gilt zu allen beobachteten Zeitpunkten und bestätigt Hypo- 
these 1a. Der positive Zusammenhang spricht zum einen dafür, dass tarifvertragliche Regelungen zu Arbeitszeitkonten und Betriebs-/Personalräte als Verhandlungspartner Transaktionskosten senken. Zum anderen spricht er dafür, dass Gewerkschaften und Betriebs-/Personalräte Arbeitszeitkonten befürworten und ihre Einführung sowie Regulierung aktiv fördern. Im Jahr 2012 haben tarifgebundene Betriebe im Durchschnitt eine um rund 6 Prozentpunkte höhere Wahrscheinlichkeit von Arbeitszeitkonten und in Betrieben mit Betriebs-/ Personalrat ist diese um rund 10 Prozentpunkte höher. Der Effekt eines existierenden Betriebs-/Personalrates ist zu allen Zeitpunkten stärker als der einer Tarifbindung. Dies kann dahingehend interpretiert werden, dass Betriebs-/Personalräte im Zuge des BetrVG eine wesentliche Rolle bei Einführung und Ausgestaltung von Arbeitszeitkonten spielen. Bei betrieblichen Vereinbarungen kann die Regulierung und Ausgestaltung von Arbeitszeitkonten besser an die unterschiedlichen betrieblichen Bedürfnisse angepasst werden und durch das Mitbestimmungsrecht der Betriebs- oder Personalräte können auch die Wünsche der Beschäftigten besser berücksichtigt werden. Dabei geben die Tarifverträge den betrieblichen Akteuren den notwendigen Spielraum, um Arbeitszeiten zu regeln. Die hohe Mitbestimmungs- und Ausgestaltungskompetenz auf der betrieblichen Ebene ist somit auch auf tarifvertragliche Regelungen zurückzuführen.

Zweitens zeigt sich überwiegend ein signifikanter Zusammenhang zwischen dem Einsatz externer Flexibilitätsinstrumente und Arbeitszeitkonten. Dabei steigt die Wahrscheinlichkeit von Arbeitszeitkonten mit einem zunehmenden Anteil an Neueinstellungen und bestätigt Hypothese 2b. Die Effekte sind aber nur gegen Ende des Beobachtungszeitraums signifikant und weisen auf ein komplementäres Verhältnis hin. So werden auch in Arbeitszeitkontenbetrieben Neueinstellungen notwendig, wenn der Arbeitseinsatz langfristig an ein höheres Arbeitsaufkommen angepasst werden muss. Dagegen zeigt sich, mit Ausnahme des Jahres 2006, kein signifikanter Zusammenhang zwischen Arbeitszeitkonten und dem Anteil an betriebsbedingten Kündigungen. Betriebsbedingte Kündigungen scheinen Arbeitszeitkonten daher nicht grundsätzlich im Wege zu stehen oder diese zu erschweren.

Tabelle 3: Determinanten von Arbeitszeitkonten - Logistische Regressionen

\begin{tabular}{|c|c|c|c|c|c|c|}
\hline & \multicolumn{2}{|l|}{2006} & \multicolumn{2}{|c|}{2009} & \multicolumn{2}{|c|}{2012} \\
\hline & AME & $\begin{array}{l}\text { Std.- } \\
\text { fehler }\end{array}$ & AME & $\begin{array}{l}\text { Std.- } \\
\text { fehler }\end{array}$ & AME & $\begin{array}{l}\text { Std.- } \\
\text { fehler }\end{array}$ \\
\hline \multicolumn{7}{|l|}{ Tarifbindung (Ref.: Kein Tarifvertrag) } \\
\hline Tarifbindung & $0,069 * * *$ & 0,013 & $0,077 * * *$ & 0,012 & $0,057 * * *$ & 0,012 \\
\hline \multicolumn{7}{|c|}{ Betriebs-/Personalrat (Ref.: Kein Betriebs-/Personalrat) } \\
\hline Betriebs-/Personalrat & $0,142 * * *$ & 0,016 & $0,097 * * *$ & 0,015 & $0,096 * * *$ & 0,015 \\
\hline Anteil der Neueinstellungen & 0,000 & 0,000 & $0,001 * * *$ & 0,000 & $0,001 * * *$ & 0,000 \\
\hline Anteil der Kündigungen durch Betrieb & $-0,003 * * *$ & 0,001 & $-0,001$ & 0,001 & 0,000 & 0,001 \\
\hline Anteil befristet Beschäftigter & 0,000 & 0,000 & $-0,001 * * *$ & 0,000 & $-0,001 * * *$ & 0,000 \\
\hline Anteil der Leiharbeiter & $0,004 * * *$ & 0,001 & $0,004 * * *$ & 0,001 & $0,003 * *$ & 0,001 \\
\hline Anteil der Teilzeitbeschäftigten & $-0,001 * * *$ & 0,000 & 0,000 & 0,000 & 0,000 & 0,000 \\
\hline Anteil geringfügig Beschäftigter & 0,000 & 0,000 & $-0,002 * * *$ & 0,000 & $-0,002 * * *$ & 0,000 \\
\hline Frauenanteil & $-0,001 * *$ & 0,000 & 0,000 & 0,000 & $-0,001 * * *$ & 0,000 \\
\hline $\begin{array}{l}\text { Anteil der Facharbeiter und Ang./Bea. mit } \\
\text { qualifizierter Tätigkeit }\end{array}$ & $0,001 * * *$ & 0,000 & $0,001 * * *$ & 0,000 & $0,001 * * *$ & 0,000 \\
\hline Logarithmierte Betriebsgröße & $0,055 * * *$ & 0,004 & $0,054 * * *$ & 0,004 & $0,064 * * *$ & 0,005 \\
\hline
\end{tabular}




\begin{tabular}{|c|c|c|c|c|c|c|}
\hline & \multicolumn{2}{|l|}{2006} & \multicolumn{2}{|c|}{2009} & \multicolumn{2}{|c|}{2012} \\
\hline & AME & $\begin{array}{l}\text { Std.- } \\
\text { fehler }\end{array}$ & AME & $\begin{array}{l}\text { Std.- } \\
\text { fehler }\end{array}$ & AME & $\begin{array}{l}\text { Std.- } \\
\text { fehler }\end{array}$ \\
\hline \multicolumn{7}{|l|}{ Sektor (Ref:: Baugewerbe) } \\
\hline Land- und Forstwirstschaft/Fischerei & $-0,013$ & 0,044 & 0,028 & 0,047 & 0,059 & 0,042 \\
\hline Bergbau/Energie- und Wasserversorgung & $-0,018$ & 0,049 & 0,025 & 0,042 & 0,068 & 0,042 \\
\hline Verbrauchsgüterindustrie/Nahrungs- & -0042 & 0031 & -0002 & 0030 & 0009 & 0030 \\
\hline $\begin{array}{l}\text { Produktionsgüterindustrie/ Grundstoffverar- } \\
\text { beitung }\end{array}$ & $-0,080 * * *$ & 0,029 & 0,019 & 0,029 & 0,041 & 0,029 \\
\hline Investitions- und Gebrauchsgüterindustrie & 0,024 & 0,027 & $0,082 * * *$ & 0,024 & $0,083 * * *$ & 0,025 \\
\hline Handel/Reparatur & $-0,139 * * *$ & 0,028 & $-0,072 * * *$ & 0,028 & $-0,057 * *$ & 0,028 \\
\hline Verkehr/Information und Kommunikation & $-0,194 * * *$ & 0,031 & $-0,109 * * *$ & 0,031 & $-0,082 * * *$ & 0,030 \\
\hline KreditNersicherungen & $-0,155 * * *$ & 0,036 & $-0,133 * * *$ & 0,041 & $-0,091 * *$ & 0,040 \\
\hline Gastgewerbe & $-0,203 * * *$ & 0,039 & $-0,064$ * & 0,036 & $-0,058$ & 0,036 \\
\hline Erziehung und Unterricht & $-0,187 * * *$ & 0,037 & $-0,123 * * *$ & 0,039 & $-0,076 * *$ & 0,036 \\
\hline Gesundheits-/Sozial- und Veterinärwesen & $-0,199 * * *$ & 0,030 & $-0,079 * *$ & 0,031 & $-0,067 * *$ & 0,031 \\
\hline Sonstige Dienstleistungen & $-0,105 * * *$ & 0,026 & $-0,025$ & 0,026 & 0,005 & 0,026 \\
\hline $\begin{array}{l}\text { Org. ohne Erwerbscharakter/Öff. Verwal- } \\
\text { tung/Sozialversicherung }\end{array}$ & $-0,087 * * *$ & 0,030 & $-0,016$ & 0,031 & 0,058 * & 0,030 \\
\hline \multicolumn{7}{|c|}{ Art des Betriebs/der Dienststelle (Ref.: Eigenständiges Unternehmen) } \\
\hline Niederlassung/Dienststelle/Filiale & 0,003 & 0,017 & $-0,012$ & 0,017 & 0,010 & 0,018 \\
\hline Zentrale/Hauptverwaltung & $0,063 * * *$ & 0,014 & $0,066 * * *$ & 0,014 & $0,080 * * *$ & 0,014 \\
\hline Regionale oder fachliche Mittelinstanz & 0,029 & 0,028 & 0,043 & 0,035 & 0,018 & 0,035 \\
\hline \multicolumn{7}{|c|}{ Technischer Stand (Ref.: Weder neue noch alte Technologie) } \\
\hline Neue Technologie & $0,035 * * *$ & 0,012 & 0,020 * & 0,012 & 0,011 & 0,011 \\
\hline Alte Technologie & $-0,001$ & 0,031 & 0,016 & 0,030 & $-0,036$ & 0,029 \\
\hline \multicolumn{7}{|l|}{ Investitionen (Ref.: Keine Investitionen) } \\
\hline $\begin{array}{l}\text { Investitionen in EDV, Informations- und Kom- } \\
\text { munikationstechnik }\end{array}$ & $0,054 * * *$ & 0,012 & $0,052 * * *$ & 0,011 & $0,033 * * *$ & 0,011 \\
\hline \multicolumn{7}{|l|}{ Region (Ref.: Westdeutschland) } \\
\hline Ostdeutschland & 0,018 & 0,012 & 0,006 & 0,011 & 0,008 & 0,011 \\
\hline Anteil nichtübernommener Beschäftigter & $-0,006 * *$ & 0,003 & $-0,007 * *$ & 0,003 & $-0,012 * * *$ & 0,003 \\
\hline $\begin{array}{l}\text { Anteil von Beschäftigten mit auslaufendem } \\
\text { Arbeitsvertrag }\end{array}$ & $-0,003 * *$ & 0,001 & $-0,002$ & 0,001 & $-0,002$ & 0,001 \\
\hline $\begin{array}{l}\text { Anteil von Beschäftigten mit einvernehmlicher } \\
\text { Aufhebung }\end{array}$ & $-0,004$ * & 0,002 & 0,000 & 0,002 & $-0,001$ & 0,002 \\
\hline Anteil von Beschäftigten in Ruhestand & $-0,009 * *$ & 0,004 & $-0,008 * *$ & 0,004 & 0,000 & 0,003 \\
\hline Anteil von Beschäftigten in Frührente & $-0,005$ & 0,004 & $-0,009 * *$ & 0,005 & $-0,004$ & 0,004 \\
\hline$\overline{\text { Fallzahl }}$ & 6992 & & 6994 & & 7100 & \\
\hline Pseudo-R² (Mc Fadden) & 0,20 & & 0,18 & & 0,19 & \\
\hline
\end{tabular}

$* \mathrm{p}<0.1, * * \mathrm{p}<0.05, * * * \mathrm{p}<0.01$

Anmerkung: $* * * / * *$ * signalisieren einen signifikant positiven bzw. negativen Zusammenhang auf dem $1 \%-$, $5 \%$ - und $10 \%$-Niveau.

Quelle: IAB-Betriebspanel, eigene Berechnungen.

Ein negativer Zusammenhang zeigt sich zwischen dem Befristungsanteil und Arbeitszeitkonten und bestätigt Hypothese 4a. Dieses Ergebnis spricht hier für einen gewissen Trade-off zwischen Maßnahmen interner und externer Flexibilität. Bei einem Anstieg der Befristungs- 
quote um 1 Prozentpunkt sinkt die Wahrscheinlichkeit von Arbeitszeitkonten im Durchschnitt um 0,1 Prozentpunkte im Jahr 2012. So kann es für Betriebe mit einer höheren Befristungsquote durchaus schwieriger sein, Arbeitszeitkonten einzusetzen und an betriebliche Bedürfnisse auszurichten. Dies kann zum einen darauf zurückzuführen sein, dass Beschäftigte mit einem zeitlich begrenztem Arbeitsvertrag weniger in der Lage sind, den Aufbau von Zeitguthaben oder -schulden an betriebliche Bedürfnisse auszurichten. Zum anderen werden externe Flexibilitätsinstrumente bei einer relativ zur Stammbelegschaft steigenden Randbelegschaft generell bedeutender. Der positive Zusammenhang zwischen dem Anteil an Leiharbeitern und Arbeitszeitkonten zeigt sich zu allen Beobachtungszeitpunkten und spricht für die These, dass Betriebe die Leiharbeit komplementär nutzen, um sowohl intern als auch extern flexibel zu sein. Dabei dürfte es sich vor allem um Betriebe mit einem hohen Flexibilitätsbedarf und sich schnell verändernden Unternehmensumwelten handeln. In diesen Betrieben kann die Leiharbeit auch als Puffer für die Stammbelegschaft eingesetzt werden, indem bei negativen Nachfrageveränderungen der Umfang der Leiharbeit reduziert wird und (Stamm-)Beschäftigte gleichzeitig Zeitguthaben abbauen. Dies deutet auf eine zunehmende Polarisierung zwischen gut geschützten Insidern auf der einen Seite und prekär Beschäftigten auf der anderen Seite hin. Damit kann Hypothese 5b gestützt werden.

Drittens bestätigen die Ergebnisse nur teilweise die vermuteten Zusammenhänge bei den Merkmalen des vertraglichen Arbeitszeitumfangs, womit Hypothese 6 nicht voll gestützt werden kann. Mit einem zunehmenden Anteil von Teilzeit- und geringfügig Beschäftigten fällt die Wahrscheinlichkeit von Arbeitszeitkonten in Betrieben geringer aus. Dabei scheint sich aber der negative Zusammenhang von der regulären Teilzeit hin zur geringfügigen Beschäftigung verschoben zu haben. Seit dem Jahr 2003 hat die geringfügige Beschäftigung einen starken Zuwachs erfahren, da die Geringfügigkeitsgrenze auf 400 Euro angehoben und damit die betriebliche Nutzung erleichtert wurde. ${ }^{11}$ Mit einem zunehmenden Anteil geringfügig Beschäftigter kann das betriebliche Interesse an Arbeitszeitkonten sinken, da Betriebe die geringfügige Beschäftigung nutzen, um kurzfristig und flexibel auf Nachfragespitzen zu reagieren. Somit können Belastungen der Beschäftigten durch (dauerhafte) transitorische Überstunden vermieden werden. Dagegen scheint der Anteil an Teilzeitbeschäftigten nicht (mehr) dafür relevant zu sein, ob es Arbeitszeitkonten gibt oder nicht. Dies spricht für die Annahme, dass eine Teilzeittätigkeit auf Wunsch der Beschäftigten erfolgt und nicht primär als Flexibilitätsinstrument zur Anpassung an Nachfrageschwankungen genutzt wird. ${ }^{12}$

Viertens weisen die Ergebnisse auf die vermuteten Zusammenhänge bei den individuellen Beschäftigtenmerkmalen und Arbeitszeitkonten hin. Arbeitszeitkonten scheinen unterdurchschnittlich häufig in Betrieben mit einem höheren Frauenanteil vorzukommen. Die Effekte sind in den Jahren 2006 und 2012 signifikant. Es kann angenommen werden, dass es bei einer höheren Frauenquote durchaus schwieriger erscheint, Arbeitszeitkonten zur Arbeitszeitflexibilisierung nach betrieblichen Bedürfnissen zu nutzen. Hypothese 7 kann insgesamt gestützt werden. Ein positiver Zusammenhang zeigt sich zwischen dem Anteil der Facharbeiter sowie qualifizierten Beschäftigten und Arbeitszeitkonten. Facharbeiter und qualifizierte Be-

11 Zum 1.1.2013 wurde die Verdienstgrenze für Minijobs um 50 Euro auf 450 Euro erhöht.

12 Da die deutliche Mehrheit der Teilzeitbeschäftigten weiblich ist (Wanger, 2011), könnten die insignifikanten Ergebnisse beim Teilzeitanteil auch darauf zurückzuführen sein, dass der Effekt schon beim Frauenanteil eingespeist ist und der Teilzeitanteil darüber hinaus keinen zusätzlichen Erklärungsbeitrag leistet. 
schäftige sind eine kritische Ressource und stehen auf dem Arbeitsmarkt nur begrenzt zur Verfügung. Deshalb sind Betriebe an langfristigen Arbeitsverhältnissen mit diesen Beschäftigten interessiert. Betriebe können langfristige Arbeitsverhältnisse erreichen, indem sie Arbeitszeitkonten einführen und damit den Forderungen der qualifizierten Beschäftigten nach Arbeitsplatzsicherheit und guten Arbeitsbedingungen nachkommen. Weiterhin verfügen Facharbeiter und qualifizierte Beschäftigte über ein höheres Maß an allgemeinem und betriebsspezifischem Humankapital. Je höher ihr Anteil an der Gesamtbelegschaft ist, desto sinnvoller sind Maßnahmen der internen Flexibilität, um dieses Humankapital im Betrieb zu halten und zu nutzen. Bei negativen Nachfrageveränderungen wird daher zunächst versucht, den Arbeitseinsatz durch einen Guthabenabbau auf Arbeitszeitkonten nach unten anzupassen und betriebsbedingte Kündigungen zu vermeiden. Dagegen kann bei positiven Auftragsveränderungen das Humankapital intensiv genutzt werden, indem die Arbeitszeit vorübergehend verlängert wird. Der positive Zusammenhang zeigt sich zu allen Beobachtungszeitpunkten und bestätigt damit Hypothese 8 .

Bei den Kontrollvariablen können bisherige empirische Befunde zum Einfluss der Betriebsgröße bestätigt werden. So zeigt sich, dass mit zunehmender Betriebsgröße die Wahrscheinlichkeit von Arbeitszeitkonten steigt. Dafür gibt es unterschiedliche Erklärungsgründe. Zum einen sind Arbeitszeitkonten für größere Betriebe günstiger und damit attraktiver als für kleinere Betriebe. Zum anderen weisen größere Betriebe einen höheren Professionalisierungsgrad bei Arbeitszeitregelungen auf als kleinere Betriebe. In größeren Betrieben gibt es häufiger formelle Arbeitszeitregelungen. Diese formellen Arbeitszeitregelungen und -modelle beinhalten Arbeitszeitkonten, sie können aber auch durch diese umgesetzt werden. So kann beispielsweise das Arbeitszeitmodell Gleitzeit über Arbeitszeitkonten verwaltet werden (Lindecke, 2015). Weiterhin ist der Kündigungsschutz in größeren Betrieben rigider als in kleineren Betrieben, wodurch häufiger auf interne Flexibilitätsinstrumente zurückgegriffen wird.

Auch bestätigen sich empirische Befunde bei den Branchen. Arbeitszeitkonten sind in den meisten Branchen des tertiären Sektors unterdurchschnittlich häufig verbreitet. Ein positiver Zusammenhang zeigt sich zwischen den getätigten Investitionen im Bereich EDV, Informations- und Kommunikationstechnik und Arbeitszeitkonten. Eine moderne Technik scheint eine wichtige Voraussetzung für ein modernes Arbeitszeitkontenerfassungssystem zu sein (Bellmann \& Gewiese, 2004). Dagegen scheint es nicht von Bedeutung zu sein, wie der technische Stand der Anlagen und Betriebs- und Geschäftsausstattung ist. Auch gibt es keine signifikanten Unterschiede zwischen ost- und westdeutschen Betrieben. Bei den zusätzlich aufgenommenen Instrumenten externer Flexibilität zeigt sich nur vereinzelt ein signifikanter Zusammenhang. So scheint mit einem steigenden Anteil nicht übernommener Beschäftigter die Wahrscheinlichkeit von Arbeitszeitkonten zu sinken. Mit diesem Instrument können Betriebe ihren Personalbedarf an die betriebliche Situation anpassen, ohne auf (teure) Entlassungen zurückgreifen zu müssen.

\section{Veränderungen über die Zeit}

Bislang wurde analysiert, welche betrieblichen Faktoren Arbeitszeitkonten begünstigen und welche sie erschweren. Bei diesen Bestimmungsfaktoren kann es zu Veränderungen über die Zeit kommen. Um zeitliche Veränderungen auf statistische Signifikanz zu prüfen wurde eine gepoolte Schätzung durchgeführt. Dabei wurde getestet, ob die marginalen Effekte 
signifikant verschieden sind, d.h. der Einfluss der unabhängigen Variable auf die Wahrscheinlichkeit von Arbeitszeitkonten sich über die Zeit ändert. ${ }^{13}$

Hinsichtlich der Tarifbindung kann die Nullhypothese nicht verworfen werden. Die marginalen Effekte sind in den Jahren 2006, 2009 und 2012 nicht signifikant verschieden, d.h. es gibt keine zeitlichen Veränderungen beim Einfluss der Tarifbindung auf die Wahrscheinlichkeit von Arbeitszeitkonten. Bei der Existenz eines Betriebs-/Personalrates zeigen sich in den Jahren 2009 und 2012 signifikant verschiedene marginale Effekte zum Jahr 2006. Diese sind in 2009 und 2012 (im Betrag) kleiner (s. Tabelle 3). Damit wird der Einfluss eines Betriebs-/ Personalrates auf die Wahrscheinlichkeit von Arbeitszeitkonten über die Zeit schwächer. Der Effekt des Anteils der Neueinstellungen ändert sich ebenfalls über die Zeit und ist in 2009 und 2012 (im Betrag) größer und damit stärker als in 2006. Beim Anteil der Kündigungen zeigt sich nur zwischen 2006 und 2012 ein signifikanter Unterschied. Über die Zeit wird der Effekt des Anteils der Kündigungen (im Betrag) kleiner. Dagegen gibt es sowohl beim Anteil der befristet Beschäftigten als auch beim Anteil der Leiharbeiter keine zeitlichen Veränderungen.

Beim Anteil der Teilzeitbeschäftigten sind die marginalen Effekte in den Jahren 2006 und 2012 signifikant verschieden, beim Anteil der geringfügig Beschäftigten zeigen sich in den Jahren 2009 und 2012 signifikante Unterschiede. Bei beiden Bestimmungsfaktoren gibt es also zeitliche Änderungen. Während bei der Teilzeitbeschäftigung der marginale Effekt (im Betrag) kleiner wird, wird er bei der geringfügigen Beschäftigung (im Betrag) größer. Beim Frauenanteil und dem Anteil der Facharbeiter und qualifizierten Beschäftigten kann die Nullhypothese jeweils nicht verworfen werden. Dies bedeutet, dass sich der Einfluss des Frauenanteils und des Anteils der Facharbeiter und qualifizierten Beschäftigten auf die Wahrscheinlichkeit von Arbeitszeitkonten nicht über die Zeit ändert.

\section{Bestimmungsfaktoren der Verbreitungsquote von Arbeitszeitkonten bei Be- schäftigten}

Tabelle 4 zeigt die Ergebnisse der OLS-Regressionen für die Partizipationsrate der Beschäftigten, d.h. die Verbreitungsquote von Arbeitszeitkonten bei Beschäftigten in Betrieben mit Arbeitszeitkonten. Bei diesen Regressionsmodellen wurden die unabhängigen Variablen ebenfalls auf Multikollinearität getestet, wobei es keine Hinweise auf ein Multikollinearitätsproblem gibt.

Die Ergebnisse für das Jahr 2012 zeigen, dass die Verbreitungsquote von Arbeitszeitkonten bei Beschäftigten in tarifgebundenen Betrieben und in Betrieben mit Betriebs-/Personalrat jeweils durchschnittlich um rund 1,8 Prozentpunkte höher ist als in nicht tarifgebundenen Betrieben und Betrieben ohne Betriebs-/Personalrat. Auffallend sind die unterschiedlichen Ergebnisse im Krisenjahr 2009. Während der Zusammenhang zwischen der Verbreitungsquote und der Tarifbindung negativ, aber statistisch nicht signifikant ist, ist er bei einem Betriebs-/Personalrat positiv. Dies weist erneut darauf hin, dass Betriebs-/Personalräte eine wichtige Rolle bei Arbeitszeitkontenregelungen spielen. So könnten sie be-

13 Es wurden ebenfalls Fixed-Effects- und Random-Effects Modelle geschätzt. Beim Hausman-Test wurde die Nullhypothese nicht verworfen. Somit gibt es keine signifikanten Unterschiede zwischen Fixed-Effects und Random-Effects Modellen, wodurch man das Random-Effects Modell bevorzugt. An dieser Stelle werden jedoch nur die Ergebnisse der gepoolten Schätzungen dargestellt, da diese Modelle im Vergleich zu RandomEffects Modellen keine strikte Exogenität der Regressoren verlangen. 
dingt durch die Krise auch versucht haben, die Arbeitszeitkontenregelungen (temporär) auf noch mehr Beschäftigte auszuweiten und die negativen Krisenfolgen weiter abzuschwächen. Dadurch fällt der Unterschied zu Betrieben ohne Betriebs-/Personalrat größer aus.

Tabelle 4: Determinanten der Verbreitungsquote von Arbeitszeitkonten bei Beschäftigten - OLS-Regressionen

\begin{tabular}{|c|c|c|c|c|c|c|}
\hline & \multicolumn{2}{|c|}{2006} & \multicolumn{2}{|c|}{2009} & \multicolumn{2}{|c|}{2012} \\
\hline & AME & $\begin{array}{l}\text { Std.- } \\
\text { fehler }\end{array}$ & AME & $\begin{array}{l}\text { Std.- } \\
\text { fehler }\end{array}$ & AME & $\begin{array}{l}\text { Std.- } \\
\text { fehler }\end{array}$ \\
\hline \multicolumn{7}{|l|}{ Tarifbindung (Ref.: Kein Tarifvertrag) } \\
\hline Tarifbindung & 1,005 & 1,079 & $-0,949$ & 0,914 & 1,778 * & 0,944 \\
\hline \multicolumn{7}{|c|}{ Betriebs-/Personalrat (Ref.: Kein Betriebs-/Personalrat) } \\
\hline Betriebs-/Personalrat & 1,848 & 1,233 & $2,708 * * *$ & 1,044 & $1,792 *$ & 1,074 \\
\hline Anteil der Neueinstellungen & $0,119 * * *$ & 0,044 & 0,037 & 0,030 & 0,065 * & 0,039 \\
\hline Anteil der Kündigungen durch Betrieb & 0,039 & 0,094 & 0,054 & 0,060 & $-0,029$ & 0,082 \\
\hline Anteil befristet Beschäftigter & $-0,115 * * *$ & 0,038 & $-0,052$ * & 0,031 & $-0,026$ & 0,032 \\
\hline Anteil der Leiharbeiter & 0,111 & 0,068 & $0,192 * * *$ & 0,067 & $0,174 * *$ & 0,075 \\
\hline Anteil der Teilzeitbeschäftigten & 0,038 & 0,030 & $-0,001$ & 0,025 & $-0,032$ & 0,025 \\
\hline Anteil geringfügig Beschäftigter & $-0,304 * * *$ & 0,050 & $-0,235 * * *$ & 0,041 & $-0,238 * * *$ & 0,042 \\
\hline Frauenanteil & $0,114 * * *$ & 0,024 & 0,035 * & 0,020 & $0,081 * * *$ & 0,021 \\
\hline $\begin{array}{l}\text { Anteil der Facharbeiter und Ang./Bea. mit } \\
\text { qualifizierter Tätigkeit }\end{array}$ & 0,020 & 0,019 & 0,005 & 0,016 & 0,024 & 0,016 \\
\hline Logarithmierte Betriebsgröße & $-1,533 * * *$ & 0,382 & $-2,153 * * *$ & 0,343 & $-2,115 * * *$ & 0,350 \\
\hline \multicolumn{7}{|l|}{ Sektor (Ref.: Baugewerbe) } \\
\hline Land- und Forstwirstschaft/Fischerei & $-6,656$ * & 4,039 & $-7,338 *$ & 3,902 & $-1,463$ & 3,771 \\
\hline Bergbau/Energie- und Wasserversorgung & 4,973 & 3,125 & 0,284 & 2,779 & $-0,295$ & 2,884 \\
\hline $\begin{array}{l}\text { Verbrauchsgüterindustrie/Nahrungs- und } \\
\text { Genussmittel }\end{array}$ & $-0,034$ & 2,453 & $-0,830$ & 2,274 & 3,037 & 2,400 \\
\hline $\begin{array}{l}\text { Produktionsgüterindustrie/ Grundstoffver- } \\
\text { arbeitung }\end{array}$ & 1,745 & 2,271 & 2,800 & 2,121 & $-0,855$ & 2,190 \\
\hline Investitions- und Gebrauchsgüterindustrie & $8,392 * * *$ & 2,100 & $4,178 * *$ & 1,897 & $5,244 * * *$ & 2,000 \\
\hline Handel/Reparatur & $-2,480$ & 2,373 & $-1,436$ & 2,150 & 1,304 & 2,242 \\
\hline Verkehr/Information und Kommunikation & $-2,823$ & 2,785 & $-1,166$ & 2,324 & 0,835 & 2,350 \\
\hline KreditNersicherungen & $-1,306$ & 2,915 & 2,410 & 2,873 & 0,566 & 2,943 \\
\hline Gastgewerbe & 2,911 & 4,187 & 1,033 & 3,133 & 5,149 & 3,225 \\
\hline Erziehung und Unterricht & $-13,839 * * *$ & 3,299 & $-11,723 * * *$ & 3,023 & $-5,171 *$ & 2,900 \\
\hline Gesundheits-/Sozial- und Veterinärwesen & $-12,592 * * *$ & 2,695 & $-1,841$ & 2,384 & $-1,028$ & 2,476 \\
\hline Sonstige Dienstleistungen & 1,411 & 2,195 & 2,927 & 2,044 & 3,375 & 2,132 \\
\hline $\begin{array}{l}\text { Org. ohne Erwerbscharakter/Öff. Verwal- } \\
\text { tung/Sozialversicherung }\end{array}$ & $-2,951$ & 2,429 & $-0,762$ & 2,256 & $-1,531$ & 2,307 \\
\hline \multicolumn{7}{|c|}{ Art des Betriebs/der Dienststelle (Ref.: Eigenständiges Unternehmen) } \\
\hline Niederlassung/Dienststelle/Filiale & $-0,903$ & 1,257 & 0,486 & 1,168 & 0,981 & 1,208 \\
\hline Zentrale/Hauptverwaltung & $3,769 * * *$ & 1,057 & $3,706 * * *$ & 0,937 & $3,408 * * *$ & 0,949 \\
\hline Regionale oder fachliche Mittelinstanz & 2,140 & 2,057 & $5,128 * *$ & 2,193 & $5,029 * *$ & 2,145 \\
\hline \multicolumn{7}{|c|}{ Technischer Stand (Ref.: Weder neue noch alte Technologie) } \\
\hline Neue Technologie & $3,878 * * *$ & 0,969 & $2,971 * * *$ & 0,871 & $1,837 * *$ & 0,848 \\
\hline Alte Technologie & 1,275 & 2,686 & 0,416 & 2,400 & $-0,396$ & 2,325 \\
\hline \multicolumn{7}{|l|}{ Investitionen (Ref.: Keine Investitionen) } \\
\hline $\begin{array}{l}\text { Investitionen in EDV, Informations- und } \\
\text { Kommunikationstechnik }\end{array}$ & $-1,378$ & 0,957 & 0,402 & 0,798 & $-1,459$ * & 0,810 \\
\hline
\end{tabular}




\begin{tabular}{|c|c|c|c|c|c|c|}
\hline & \multicolumn{2}{|c|}{2006} & \multicolumn{2}{|c|}{2009} & \multicolumn{2}{|c|}{2012} \\
\hline & AME & $\begin{array}{l}\text { Std.- } \\
\text { fehler }\end{array}$ & AME & $\begin{array}{l}\text { Std.- } \\
\text { fehler }\end{array}$ & AME & $\begin{array}{l}\text { Std.- } \\
\text { fehler }\end{array}$ \\
\hline \multicolumn{7}{|l|}{ Region (Ref.: Westdeutschland) } \\
\hline Ostdeutschland & $-1,189$ & 0,949 & $-1,860 * *$ & 0,818 & $-1,939 * *$ & 0,837 \\
\hline Anteil nichtübernommener Beschäftigter & $-0,777 * *$ & 0,341 & 0,182 & 0,296 & $-1,021 * * *$ & 0,379 \\
\hline $\begin{array}{l}\text { Anteil von Beschäftigten mit auslaufendem } \\
\text { Arbeitsvertrag }\end{array}$ & $-0,189$ & 0,140 & 0,171 * & 0,099 & $-0,080$ & 0,144 \\
\hline $\begin{array}{l}\text { Anteil von Beschäftigten mit einvernehmli- } \\
\text { cher Aufhebung }\end{array}$ & $-0,013$ & 0,298 & 0,130 & 0,139 & $-0,001$ & 0,175 \\
\hline Anteil von Beschäftigten in Ruhestand & 0,343 & 0,415 & $-0,431$ & 0,404 & $-0,158$ & 0,287 \\
\hline Anteil von Beschäftigten in Frührente & $-0,187$ & 0,454 & $-0,091$ & 0,433 & $-0,223$ & 0,415 \\
\hline Fallzahl & 3954 & & 4501 & & 4276 & \\
\hline $\mathrm{R}^{2}$ (Mc Fadden) & 0,07 & & 0,05 & & 0,04 & \\
\hline
\end{tabular}

$* \mathrm{p}<0.1, * * \mathrm{p}<0.05, * * * \mathrm{p}<0.01$

Anmerkung: $* * * / * * / *$ signalisieren einen signifikant positiven bzw. negativen Zusammenhang auf dem $1 \%-$, $5 \%$ - und $10 \%$-Niveau.

Quelle: IAB-Betriebspanel, eigene Berechnungen.

Beim Anteil der Neueinstellungen zeigt sich wie bei der Verbreitung von Arbeitszeitkonten in Betrieben auch bei der Verbreitungsquote bei Beschäftigten ein positiver Zusammenhang, während dieser beim Anteil der Kündigungen im Jahr 2012 negativ, aber statistisch nicht signifikant ist. Der positive Zusammenhang zwischen dem Anteil der Leiharbeiter und der Verbreitungsquote von Arbeitszeitkonten bei Beschäftigten weist erneut auf die komplementäre Nutzung der beiden Instrumente hin. Je höher der Anteil der Leiharbeiter, desto höher ist auch die Verbreitungsquote bei Beschäftigten. Dagegen hat der Anteil der Teilzeitbeschäftigten keinen Einfluss auf die Verbreitungsquote, beim Anteil der geringfügig Beschäftigten zeigt sich ein negativer Zusammenhang. Mit einem zunehmenden Anteil geringfügig Beschäftigter fällt also zum einen generell die Wahrscheinlichkeit von Arbeitszeitkonten in Betrieben geringer aus, zum anderen sinkt auch die Verbreitungsquote von Arbeitszeitkonten bei Beschäftigten in Betrieben mit Arbeitszeitkonten. Steigt der Anteil geringfügig Beschäftigter um 1 Prozentpunkt so sinkt im Durchschnitt die Verbreitungsquote bei Beschäftigten um 0,2 Prozentpunkte im Jahr 2012.

Während Arbeitszeitkonten unterdurchschnittlich häufig in Betrieben mit einem höheren Frauenanteil vorkommen, steigt die Verbreitungsquote von Arbeitszeitkonten bei Beschäftigten mit einem höheren Frauenanteil. Sofern Betriebe Arbeitszeitkonten einführen, hat der Frauenanteil einen positiven Einfluss auf die Verbreitungsquote. Dagegen zeigt sich kein signifikanter Zusammenhang zwischen dem Anteil der Facharbeiter und qualifizierten Beschäftigten und der Verbreitungsquote von Arbeitszeitkonten bei Beschäftigten.

\section{Veränderungen über die Zeit}

Bei den Bestimmungsfaktoren der Verbreitungsquote von Arbeitszeitkonten bei Beschäftigten in Betrieben mit Arbeitszeitkonten wurde ebenfalls geprüft, ob sich die marginalen Effekte signifikant voneinander unterscheiden und es damit Veränderungen über die Zeit gibt.

Bei der Tarifbindung sind die marginalen Effekte nur in den Jahren 2009 und 2012 signifikant verschieden, wobei der Effekt in 2012 (im Betrag) größer ist. Hinsichtlich der Existenz 
eines Betriebs-/Personalrates kann die Nullhypothese nicht verworfen werden, d. h. der Einfluss eines Betriebs-/Personalrates auf die Verbreitungsquote von Arbeitszeitkonten bei Beschäftigten ändert sich nicht über die Zeit. Beim Anteil der Neueinstellungen sind die marginalen Effekte in den Jahren 2006 und 2009 nur auf dem 10\%-Niveau signifikant verschieden. Dagegen gibt es beim Anteil der Kündigungen keine signifikanten Unterschiede und damit keine Veränderungen im Zeitverlauf. Beim Anteil der befristet Beschäftigten sind die marginalen Effekte in 2006 und 2012 auf dem 10\%-Niveau signifikant verschieden. Beim Anteil der Leiharbeiter gibt es erneut keine signifikanten Unterschiede, wodurch es keine Veränderungen im Zeitverlauf gibt.

Wie beim Anteil befristet Beschäftigter wird auch beim Anteil der Teilzeitbeschäftigten die Nullhypothese nur auf dem 10\%-Niveau verworfen. Dabei sind die marginalen Effekte in den Jahren 2006 und 2012 signifikant verschieden. Beim Anteil der geringfügig Beschäftigten gibt es keine signifikanten Unterschiede und damit keine zeitlichen Veränderungen. Beim Frauenanteil zeigen sich signifikant verschiedene marginale Effekte zwischen 2006 und 2009 und zwischen 2009 und 2012, wobei bei letzterem Vergleich die Nullhypothese nur auf dem 10\%-Niveau verworfen werden kann. Dagegen ändert sich der Einfluss des Anteils der Facharbeiter und qualifizierten Beschäftigten auf die Verbreitungsquote von Arbeitszeitkonten bei Beschäftigten nicht über die Zeit.

\section{Fazit}

Arbeitszeitkonten sind in Deutschland inzwischen weit verbreitet, um Arbeitszeiten zu flexibilisieren und Schwankungen abzufedern. Mit ihrer Verbreitung steht auch die Zunahme von transitorischen Überstunden im Zusammenhang, die auf Arbeitszeitkonten verwaltet werden können. Ziel des vorliegenden Beitrags war es, die Verbreitung und die Bestimmungsfaktoren von Arbeitszeitkonten in Betrieben zu analysieren und dabei zeitliche Entwicklungen aufzuzeigen. Ergänzend dazu wurde auch die Verbreitungsquote von Arbeitszeitkonten bei Beschäftigten in Betrieben mit Arbeitszeitkonten analysiert und so der Blick darauf geweitet.

Die Ergebnisse zeigen, dass Arbeitszeitkonten überdurchschnittlich häufig in tarifgebundenen und in Betrieben mit Betriebs-/Personalrat eingesetzt werden. Mit einem zunehmenden Anteil an Leiharbeitern und Facharbeitern/qualifizierten Beschäftigten steigt ebenfalls die Wahrscheinlichkeit von Arbeitszeitkonten. Dagegen fällt sie mit einem zunehmenden Anteil an befristet Beschäftigten und Frauen geringer aus. Zwar zählen sowohl die Leiharbeit als auch Befristungen zu den Instrumenten externer Flexibilität, der Richtungszusammenhang mit Arbeitszeitkonten fällt aber unterschiedlich aus. Dies spricht dafür, dass Befristungen und Leiharbeit in unterschiedlichen Teilsegmenten des Arbeitsmarktes mit unterschiedlichen Funktionen eingesetzt werden. Insgesamt weisen die genannten Bestimmungsfaktoren trotz unterschiedlicher konjunktureller Lagen eine hohe zeitliche Konstanz auf, ihr Einfluss auf die Wahrscheinlichkeit von Arbeitszeitkonten ändert sich - mit Ausnahme bei einem Betriebs-/Personalrat - nicht. Daher kann angenommen werden, dass diese Bestimmungsfaktoren auch in Zukunft gelten. Dagegen zeigen sich bei den anderen Bestimmungsfaktoren zeitliche Veränderungen. So gewinnt der Anteil der Neueinstellungen gegen Ende des Beobachtungszeitraums an Bedeutung und es scheint sich eine Effektverschiebung von der Teilzeit- zur geringfügigen Beschäftigung zu zeigen. Letzteres spricht 
dafür, dass Betriebe geringfügige Beschäftigungsverhältnisse substitutiv zu Arbeitszeitkonten einsetzen können, um betriebliche Flexibilität zu gewährleisten. Dagegen scheinen Teilzeitbeschäftigungen diese Funktion nicht (mehr) zu erfüllen. Sie werden vielmehr in Betrieben angeboten, um dem Wunsch der Beschäftigten nach kürzeren Arbeitszeiten nachzukommen und z.B. Beruf und Familie besser zu vereinbaren.

Die weiteren Befunde zeigen einen positiven Zusammenhang zwischen einem Betriebs-/ Personalrat sowie dem Anteil der Neueinstellungen und der Verbreitungsquote von Arbeitszeitkonten bei Beschäftigten. Die Verbreitungsquote steigt ebenfalls mit einem zunehmenden Anteil an Leiharbeitern und Frauen. Mit einem zunehmenden Anteil befristet und geringfügig Beschäftigter sinkt dagegen die Verbreitungsquote von Arbeitszeitkonten bei Beschäftigten. Dabei weisen ein Betriebs-/Personalrat, der Anteil an Kündigungen und Leiharbeitern sowie der Anteil an geringfügig Beschäftigten und Facharbeitern/qualifizierten Beschäftigten eine hohe zeitliche Konstanz auf, denn ihr Einfluss zeigt keine zeitlichen Veränderungen.

In den aktuellen Arbeitszeitdebatten und Kampagnen von Gewerkschaften werden die Ausgestaltungsmöglichkeiten und Funktionen von Arbeitszeitkonten (wieder) stärker in den Blickpunkt gerückt. So fordern die Gewerkschaften IG Metall und Ver.di selbstbestimmtere und an den Interessen und Lebensbedürfnissen der Beschäftigten ausgerichtete Arbeitszeiten. In Verbindung damit steht auch die Forderung, dass jede geleistete Arbeitsstunde erfasst und längere Arbeitszeiten durch Freizeitausgleich wieder abgegolten werden. Die Gewerkschaften sehen dabei Langzeit- oder Lebensarbeitszeitkonten als wichtiges Instrument, um zum einen biografieorientierte Arbeitszeiten für Beschäftigte zu realisieren und zum anderen einem Verfall von Arbeitszeiten zu begegnen. Biografieorientierte Arbeitszeiten sollen es Beschäftigten ermöglichen ihre Arbeitszeiten besser mit ihrer persönlichen Lebenssituation zu vereinbaren und Auszeiten für beispielsweise Weiterbildung, Sabbatical oder Pflegeaufgaben zu realisieren. So sollen verlässliche kollektive Rahmenregelungen Entscheidungsmöglichkeiten für Beschäftigte bieten, um eine individuelle Arbeitszeitgestaltung im Lebensverlauf zu realisieren.

Zwar werden Langzeit- und Lebensarbeitszeitkonten gesetzlich geregelt, dennoch gibt es in der betrieblichen Praxis weiter Unsicherheiten und Risiken. Es bedarf institutioneller Arrangements, die verbindliche Nutzungsansprüche bei Langzeitkonten definieren, Vertrauen zwischen den Betriebsparteien begründen, den Umgang mit Interessenkonflikten regeln und eine langfristige Beschäftigungsperspektive stärken. Dabei können tarifliche Regelungen zu Langzeitkonten einen wichtigen Beitrag leisten (Wotschack, 2012). Gewerkschaften haben aber auch die Aufgabe betriebliche Interessenvertreter zu unterstützen, um aufbauend auf den kollektiven Rahmenregelungen passgenaue betriebliche Vereinbarungen zu realisieren.

Den Kampagnen und Forderungen der Gewerkschaften nach mehr Zeitsouveränität für die Beschäftigten stehen die Forderungen der Arbeitgeber nach (noch) mehr Flexibilität der Beschäftigten aufgrund betrieblicher Erfordernisse gegenüber. Vor dem Hintergrund eines zunehmenden Wettbewerbs um qualifizierte Arbeitskräfte ist jedoch eine stärkere Regulierung und Ausgestaltung von Arbeitszeitkonten nach individuellen Bedürfnissen auch für Arbeitgeber interessant und vorteilhaft, um die Attraktivität als Arbeitgeber zu steigern und qualifizierte Arbeitskräfte langfristig an den Betrieb zu binden. Dennoch sind auch betriebliche Belange zu berücksichtigen, um bei Nachfrageschwankungen weiterhin den Arbeitseinsatz an das Arbeitsaufkommen anpassen zu können. Den Gewerkschaften und Arbeitgeberverbänden auf kollektiver Ebene und den Betriebs-/Personalräten und Arbeitgebern auf 
betrieblicher Ebene obliegt es gemeinsam an Lösungen zu arbeiten, damit Arbeitszeitkonten (weiterhin) sowohl für Arbeitgeber als auch für Arbeitnehmer attraktiv sind.

\section{Literatur}

Allan, C., Brosnan, P. \& Walsh, P. (1998). Non standard working time arrangements in Australia and New Zealand. International Journal of Manpower, 19 (4), 234-249. doi: $10.1108 / 01437729810220419$

Auspurg, K. \& Hinz, T. (2011). Gruppenvergleiche bei Regressionen mit binären abhängigen Variablen. Probleme und Fehleinschätzungen am Beispiel von Bildungschancen im Kohortenverlauf. Zeitschrift für Soziologie, 40 (1), 62-73. doi: 10.1515/zfsoz-2011-0104

Bäcker, G. (2006). Was heißt hier „geringfügig“? - Minijobs als wachsendes Segment prekärer Beschäftigung. WSI Mitteilungen, 59 (5), 255-262.

Balleer, A., Gehrke, B. \& Merkl, C. (2017). Some surprising facts about working time accounts and the business cycle in Germany. International Journal of Manpower, 38 (7), 940-953. doi: 10.1108/IJM-05-2017-0100

Bauer, F., Groß, H., Munz, E. \& Sayin, S. (2002). Arbeits und Betriebszeiten 2001. Neue Formen des betrieblichen Arbeits und Betriebszeitmanagements. Ergebnisse einer repräsentativen Betriebsbefragung. Köln.

Bellmann, L. (2002). Das IAB Betriebspanel: Konzeption und Anwendungsbereiche. Allgemeines Statistisches Archiv, 86, 177-188. doi: 10.1007/s101820200094

Bellmann, L. \& Gewiese, T. (2003). Betriebliche Arbeitszeitstrukturen in der Bundesrepublik Deutschland. Empirische Ergebnisse aus dem IAB-Betriebspanel 1996-1999 unter besonderer Berücksichtigung der Arbeitszeitflexibilisierung. Schriftenreihe der Bundesanstalt für Arbeitsschutz und Arbeitsmedizin. Forschung, 1007. Bremerhaven: Wirtschaftsverlag NW, Verlag für neue Wissenschaft.

Bellmann, L. \& Alda, H. (2004). Betriebliche Personal und Beschäftigungspolitik zwischen Flexibilität und Stabilität. WSI Mitteilungen, 57 (5), 255-261.

Bellmann, L. \& Gewiese, T. (2004). Die Dynamik der Nutzung von Arbeitszeitkonten: Theoretische Ansätze und Längsschnittanalysen mit dem IAB Betriebspanel. In L. Bellmann \& C. Schnabel (Hrsg.), Betriebliche Arbeitszeitpolitik im Wandel. Beiträge zur Arbeitsmarkt und Berufsforschung, 288, 139-184). Nürnberg.

Bellmann, L. \& Gerner, H. D. (2011). Reversed roles? Wage and employment effects of the current crisis. In H. Immervoll, A. Peichl \& K. Tatsiramos (Hrsg.), Who loses in the downturn? Economic crisis, employment and income distribution (S. 181-206). Bingley: Emerald. doi: 10.1108/S0147-9121(2011)0000032009

Bellmann, L., Gerner, H. D. \& Upward, R. (2012). The response of German establishments to the 20082009 economic crisis. OECD Social, Employment and Migration Working Papers, 137. doi: $10.1787 / 5 \mathrm{k} 8 \times 7 \mathrm{gwmb} 3 \mathrm{jc}-\mathrm{en}$

Bellmann, L., \& Hübler, O. (2015). Working time accounts and firm performance in Germany. IZA Journal of European Labour Studies, 4, 1-18. doi.org/10.1186/s40174-015-0048-8

Berg, P. (2008). Working time flexibility in the German employment relations system: Implications for Germany and lessons for the United States. Industrielle Beziehungen, 15 (2), 133-150.

Bispinck, R. (2005). Immer flexibler - und immer länger? Tarifliche Regelungen zur Arbeitszeit und ihrer Gestaltung. Eine Analyse von 24 Tarifbereichen. Abgerufen von https://www.boeckler.de/pdf/p_ta_elemente_57_2005.pdf

Bispinck, R. (2014): Tarifliche Arbeitszeitregelungen zwischen betrieblichen Flexi Ansprüchen und individuellen Arbeitszeitoptionen. Eine Analyse ausgewählter Tarifbereiche. Abgerufen von https://www.boeckler.de/pdf/p_ta_elemente_79_2014.pdf 
Boeri, T. \& Brücker, H. (2011). Short time work benefits revisited. Some lessons from the Great Recession. Economic Policy, 26 (68), 697-765. doi: 10.1111/j.1468-0327.2011.271.x

Bosch, A., Ellguth, P. \& Promberger, M. (1992). Gleitzeit: Wieviel Zeitautonomie ist gefragt? WSI Mitteilungen, 45 (1), 51-59.

Bosch, G. (1996). Jahresarbeitszeiten - Flexibilität auch für Stammbelegschaften. Journal für Sozialforschung, 36 (1), 3-22.

Bosch, G. (2011). The German labour market after the financial crisis: Miracle or just a good policy mix? In D. Vaughan-Whitehead (Hrsg.), Work inequalities in the crisis? Evidence from Europe (S. 243-277). Cheltenham: Elgar.

Brunner, M. (2009). Ressource Dependence Ansatz. In M. Schwaiger \& A. Meyer (Hrsg.), Theorien und Methoden der Betriebswirtschaft. Handbuch für Wissenschaftler und Studierende (S. 2940). München: Vahlen.

Buis, M.L. (2010). Interpretation of interactions in nonlinear models. The Stata Journal, 10 (2), 305-308.

Bundesmann-Jansen, J., Groß, H. \& Munz, E. (2000). Arbeitszeit '99. Ergebnisse einer repräsentativen Beschäftigtenbefragung zu traditionellen und neuen Arbeitszeitformen in der Bundesrepublik Deutschland. Köln.

Burda, M. C. \& Hunt, J. (2011). What explains the German labor market miracle in the Great Recession? Brookings Papers on Economic Activity, 42 (1), 273-335. doi: org/10.1353/eca.2011.0004

Carstensen, V. (1999). Alternative Flexibilisierungsstrategien der Beschäftigung. Abgerufen von https://www.econstor.eu/bitstream/10419/78312/1/dp-229.pdf

Coase, R. H. (1960). The problem of social cost. Journal of Law and Economics, 3, 1-44. doi: $10.1086 / 466560$

Dietl, H. (2007). Transaktionskostentheorie. In R. Köhler, H.-U. Küpper \& A. Pfingsten (Hrsg.), Handwörterbuch der Betriebswirtschaft (S. 1750-1760). Stuttgart: Schäffer-Poeschel.

Ellguth, P., Gerner, H. D. \& Zapf, I. (2013). Flexibilität für Betriebe und Beschäftigte. Vielfalt und Dynamik bei den Arbeitszeitkonten. Abgerufen von http://doku.iab.de/kurzber/2013/kb0313.pdf

Ellguth, P. \& Promberger, M. (2004). Arbeitszeitsituation und Betriebsrat - eine Matched Pair Analyse mit Daten des IAB-Betriebspanels. In L. Bellmann \& C. Schnabel (Hrsg.), Betriebliche Arbeitszeitpolitik im Wandel (Beiträge zur Arbeitsmarkt und Berufsforschung, 288, S. 111-131). Nürnberg.

Engfer, U. (1982). Arbeitszeitflexibilisierung als Rationalisierungsstrategie im Dienstleistungssektor. In C. Offe, K. Hinrichs \& H. Wiesenthal (Hrsg.), Arbeitszeitpolitik. Formen und Folgen einer Neuverteilung (S. 106-115). Frankfurt am Main/New York: Campus Verlag.

Erlinghagen, M. (2005). Entlassungen und Beschäftigungssicherheit im Zeitverlauf. Zur Entwicklung unfreiwilliger Arbeitsmarktmobilität in Deutschland. Zeitschrift für Soziologie, 34 (2), 147-168.

Fischer, G., Janik, F., Müller, D. \& Schmucker, A. (2009). The IAB Establishment Panel - Things users should know. Schmollers Jahrbuch, 129, 133-148. doi: 10.3790/schm.129.1.133

Gerner, H.-D. (2012). Die Produktivitätsentwicklung und die Rolle von Arbeitszeitkonten während der Großen Rezession 2008/2009. Ergebnisse auf der Grundlage des IAB Betriebspanels. Zeitschrift für Personalforschung, 26 (1), 30-47.

Giesecke, J. (2006). Arbeitsmarktflexibilisierung und soziale Ungleichheit. Sozio ökonomische Konsequenzen befristeter Beschäftigungsverhältnisse in Deutschland und Großbritannien. Wiesbaden: VS Verlag für Sozialwissenschaften.

Goudswaard, A., Oeij, P., Brugman, T. \& de Jong, T. (2009). Good practice guide to internal flexibility policies in companies. Abgerufen von https://www.eurofound.europa.eu/sites/default/files/ef_publication/field_ef_document/ef0919en.pdf

Goudswaard, A., Dhondt, S., Vergeer, R., Oeij, P., Leede, J., Adrichem, K., Csizmadia, P., Mako, C., Illesy, M. \& Thoth, A. (2012): Organisation of working time. Implications for productivity and working conditions. Overview report. Abgerufen von 
https://www.eurofound.europa.eu/sites/default/files/ef_files/pubdocs/2012/50/en/2/EF1250EN.pdf

Greene, W. H. (2008). Econometric analysis. Upper Saddle River u.a.: Pearson Prentice Hall.

Gregory, A. \& Milner, S. (2009). Editorial: Work life balance: A matter of choice? Gender, Work and Organization, 16, 1-13. doi: 10.1111/j.1468-0432.2008.00429.x

Groß, H. \& Schwarz, M. (2006). Betriebs und Arbeitszeiten 2005. Ergebnisse einer repräsentativen Betriebsbefragung. Abgerufen von http://www.ssoar.info/ssoar/handle/document/36721

Groß, H. \& Schwarz, M. (2010). Arbeitszeit, Altersstrukturen und Corporate Social Responsibility. Eine repräsentative Betriebsbefragung. Wiesbaden: VS Verlag für Sozialwissenschaften. doi: org/10.1007/978-3-531-92121-1

Haller, P. \& Jahn, E. (2014). Zeitarbeit in Deutschland: Hohe Dynamik und kurze Beschäftigungsdauern. Abgerufen von http://doku.iab.de/kurzber/2014/kb1314.pdf

Herrmann, C., Promberger, M., Singer, S. \& Trinczek, R. (1999). Forcierte Arbeitszeitflexibilisierung. Die 35 Stunden Woche in der betrieblichen und gewerkschaftlichen Praxis. Berlin: Edition Sigma.

Herzog-Stein, A. \& Seifert, H. (2010). Der Arbeitsmarkt in der Großen Rezession - Bewährte Strategien in neuen Formen. WSI Mitteilungen, 63 (11), 1-10.

Herzog-Stein, A. \& Zapf I. (2014). Navigating the Great Recession: The impact of working time accounts in Germany. Industrial \& Labor Relations Review, 67 (3), 891-925. doi: $10.1177 / 0019793914537455$

Hildebrandt, E. (2006). Balance between work and life - New corporate impositions through flexible working time or opportunity for time sovereignty? European Societies, 8 (2), 251-271. doi: 10.1080/14616690600645001

Hildebrandt, E. (2007). Langzeitkonten, Lebensplanung und Zeithandeln. In E. Hildebrandt (Hrsg.), Lebenslaufpolitik im Betrieb. Optionen zur Gestaltung der Lebensarbeitszeit durch Langzeitkonten (S. 173-202). Berlin: Edition Sigma.

Hill, E. J., Hawkins, A. J., Ferris, M. \& Weitzman, M. (2001). Finding an extra day a week: The positive influence of perceived job flexibility on work and family life balance. Family Relations, 50 (1), 49-58. doi: 10.1111/j.1741-3729.2001.00049.x

Hinrichs, K. (1992). Zur Zukunft der Arbeitszeitflexibilisierung. Arbeitnehmerpräferenzen, betriebliche Interessen und Beschäftigungswirkungen. Soziale Welt, 43 (3), 313-330.

Hohendanner, C., Ostmeier, E. \& Ramos Lobato, P. (2015). Befristete Beschäftigung im öffentlichen Dienst. Entwicklungen, Motive und rechtliche Umsetzung. Abgerufen von http://doku.iab.de/forschungsbericht/2015/fb1215.pdf

Hohendanner, C. \& Ramos Lobato, P. (2017). Die personalpolitische Funktion befristeter Beschäftigung im öffentlichen Dienst. WSI Mitteilungen, 70 (1), 45-53.

Hunt, J. (2013). Flexible work time in Germany: Do workers like it and how have employers exploited it over the cycle? Perspektiven der Wirtschaftspolitik, 14 (1-2), 67-98.

IG Metall (2017). Kampagne „Mein Leben - meine Zeit“: Worum es geht. Abgerufen von https://www.igmetall.de/kampagne-mein-leben-meine-zeit-hintergrund-22655.htm

Kümmerling, A. \& Lehndorff, S. (2014). The use of working time related crisis response measures during the Great Recession. Abgerufen von

http://www.ilo.org/wcmsp5/groups/public/---ed_protect/---protrav/--travail/documents/publication/wcms_236369.pdf

Lindecke, C. (2015). Wem gehört die Zeit? Flexible Arbeitszeiten: Grenzenlose Freiheit oder grenzenlose Erreichbarkeit? Zeitschrift für Arbeitswissenschaft, 69 (1), 31-38. doi.org/10.1007/BF03373934

Looise, J. C., Van Riemsdijk, M. \& De Lange, F. (1998). Company labour flexibility strategies in the Netherlands: An institutional perspective. Employee Relations, 20 (5), 461-482. doi: $10.1108 / 01425459810238747$ 
Lott, Y. (2014). Working-time flexibility and autonomy: A European perspective on time adequacy. European Journal of Industrial Relations, 21 (3), 259-274. doi: 10.1177/0959680114543604

Ludewig, O. (2001). Betriebliche Bestimmungsgründe von Arbeitszeitkonten. Mitteilungen aus der Arbeitsmarkt und Berufsforschung, 34 (3), 302-313.

Munz, E., Bauer, F. \& Groß, H. (2002). Regelung und Praxis von Arbeitszeitkonten. WSI Mitteilungen, 55 (6), 334-340.

Pearce, J. (1982). The company mission statement as a strategic tool. Sloan Management Review, 23 (3), 15-24.

Pfeffer, J. \& Salancik, G. R. (1978). The external control of organisations. A resource dependence perspective. New York: Harper \& Row.

Picot, A. (1982). Transaktionskostenansatz in der Organisationstheorie. Stand der Diskussion und Aussagewert. Die Betriebswirtschaft, 42, 267-284.

Promberger, M. (2005). Wie neuartig sind flexible Arbeitszeiten? Historische Grundlinien der Arbeitszeitpolitik. In H. Seifert (Hrsg.), Flexible Zeiten in der Arbeitswelt (S. 9-39). Frankfurt am Main: Campus Verlag.

Promberger, M., Böhm, S., Heyder, T., Pamer, S. \& Strauß, K. (2002). Hochflexible Arbeitszeiten in der Industrie. Chancen, Risiken und Grenzen für Beschäftigte. Berlin: Edition Sigma.

Riedmann, A., Van Gyes, G., Román, A., Kerkhofs, M. \& Bechmann, S. (2010). European company survey 2009. Overview. Abgerufen von https://www.eurofound.europa.eu/sites/default/files/ef_files/pubdocs/2010/05/en/1/EF1005EN.pdf

Seifert, H. (1996). Arbeitszeitkonten - Modelle für mehr Zeitsouveränität oder absatzorientiertes Zeitmanagement? WSI Mitteilungen, 49 (7), 442-449.

Seifert, H. (2001). Zeitkonten: Von der Normalarbeitszeit zu kontrollierter Flexibilität. WSI Mitteilungen, 54 (2), 334-340.

Seifert, H. (2003). Präventive Arbeitsmarktpolitik und betriebliche Anpassungsflexibilität - Neue Impulse durch die Hartz-Kommission. WSI Mitteilungen, 56 (5), 284-290.

Seifert, H. (2005). Vom Gleitzeit zum Langzeitkonto. WSI Mitteilungen, 58 (6), 308-313.

Seifert, H., Kümmerling, A. \& Riedmann, A. (2013). Langzeitkonten - überschätzte Erwartungen einer biografieorientierten Zeitpolitik? WSI Mitteilungen, 66 (2), 133-143.

Sheppard, J. P. (1995). A resource dependence approach to organizational failure. Social Science Research, 24 (1), 28-62. doi: 10.1006/ssre.1995.1002

Spence, M. (1973). Job market signaling. The Quarterly Journal of Economics, 87 (3), 355-374. doi: $10.2307 / 1882010$

Vereinte Dienstleistungsgewerkschaft (ver.di). (2017). Tarifpolitische Themen. Arbeitszeit-Debatte. Abgerufen von https://www.verdi.de/wegweiser/tarifpolitik/++co++cf7e8638-467f-11e5-b5e6-5254008a33df

Vereinte Dienstleistungsgewerkschaft (ver.di). (2017). Tarifpolitische Themen. Lebenslauforientierte Arbeitszeit. Abgerufen von https://www.verdi.de/wegweiser/tarifpolitik/themen/arbeitszeit/++co++b20a14ae-cb9d-11e3a5db-52540059119e

Voss, D. \& Weinkopf, C. (2012). Niedriglohnfalle Minijob. WSI Mitteilungen, 65 (1), 5-12.

Wanger, S. (2011). Ungenutzte Potenziale in der Teilzeit. Viele Frauen würden gerne länger arbeiten. Abgerufen von http://doku.iab.de/kurzber/2011/kb0911.pdf

Wotschack, P. (2012). Keine Zeit für die Auszeit. Lebensarbeitszeit als Aspekt sozialer Ungleichheit. Soziale Welt, 63 (1), 25-44. doi: 10.5771/0038-6073-2012-1-25

Zapf, I. (2016). Bestimmungsfaktoren von Arbeitszeitkonten bei Betrieben und Beschäftigten. Sozialpolitik.Ch, 2, 1-36. 


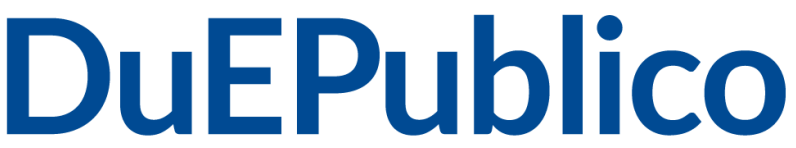

Duisburg-Essen Publications online
Offen im Denken

Ub | $\begin{aligned} & \text { universitäts } \\ & \text { bibliothek }\end{aligned}$

Dieser Text wird über DuEPublico, dem Dokumenten- und Publikationsserver der Universität Duisburg-Essen, zur Verfügung gestellt. Die hier veröffentlichte Version der EPublikation kann von einer eventuell ebenfalls veröffentlichten Verlagsversion abweichen.

DOI: $\quad 10.3224 /$ indbez.v25i1.03

URN: urn:nbn:de:hbz:464-20200702-145004-4

Dieses Werk kann unter einer Creative Commons Namensnennung

- Weitergabe unter gleichen Bedingungen 4.0 Lizenz (CC BY-SA 4.0) genutzt werden. 